\title{
Aplicação de células inativas, Compressed Sparse Row e OpenMP na simulação numérica paralelizada de reservatórios de petróleo do tipo anticlinal
}

\section{Rafael Machado de Salles ${ }^{1}$, Leonardo Figueira Werneck ${ }^{2}$, Grazione de Souza ${ }^{3}$ and Helio Pedro Amaral Souto 3}

\author{
${ }^{1}$ Engenharia Mecânica, Instituto Politécnico, UERJ, Brasil and ${ }^{2}$ Pós-graduação em Modelagem Computacional, \\ Instituto Politécnico, UERJ, Brasil and ${ }^{3}$ Departamento de Modelagem Computacional, Instituto Politécnico, \\ UERJ, Brasil. \\ rafaelmachadosalles@gmail.com; leonardofwerneck@gmail.com; *gsouza@iprj.uerj.br; helio@iprj.uerj.br
}

Submetido: 26/04/2018. Revisado: 06/06/2018. Aceito: 29/06/2018.

\begin{abstract}
Resumo
Este trabalho visa, principalmente, à simulação numérica de escoamentos monofásicos de óleo em reservatórios de petróleo do tipo anticlinal. Portanto, uma técnica específica para a representação de células inativas foi desenvolvida. Além disso, a fim de melhorar a eficiência computacional, a interface de programação OpenMP foi utilizada, juntamente com a técnica Compressed Sparse Row, com a finalidade de paralelizar-se o método dos Gradientes Conjugados, empregado na resolução do sistema algébrico de equações oriundo da discretização da Equação da Difusividade Hidráulica (EDH) que governa o escoamento. Testes de sensibilidade, convergência e desempenho foram realizados considerando-se diferentes reservatórios do tipo anticlinal.
\end{abstract}

Palavras-Chave: Anticlinal; Células inativas; Compressed Sparse Row; OpenMP; Reservatórios de petróleo

\begin{abstract}
This work aims mainly to the numerical simulation of single-phase oil flows in anticlinal reservoirs. Therefore, it was developed a specific technique for representing inactive cells. Furthermore, in order to improve the computational efficiency the OpenMP programming interface was used, along with the Compressed Sparse Row technique, to parallelize the Conjugate Gradient method employed to solve the algebraic system of equations derived from the discretization of the Hydraulic Diffusivity Equation that governs the flow. Tests of sensitivity, convergence, and performance were performed considering different anticlinal reservoirs.
\end{abstract}

Key words: Anticlinal; Compressed Sparse Row; Inactive cells; Oil reservois; OpenMP

\section{Introdução}

No século XXI, o petróleo segue sendo um insumo importante na matriz energética mundial e este cenário deve perdurar por mais algumas décadas segundo estimativas recentes, mesmo diante do aumento da utilização de outras fontes energéticas alternativas, o que é necessário devido, por exemplo, à questão ambiental por causa da poluição provocada pela combustão dos combustíveis fósseis. A escala de utilização do petróleo ainda é muito grande em função do estágio atual da sua dependência. Portanto, a indústria de óleo e gás segue tendo representatividade, no mundo e no Brasil, e vem desenvolvendo novas tecnologias de exploração e trabalhando na elaboração de estratégias para a minimização dos riscos e a maximização da sua 


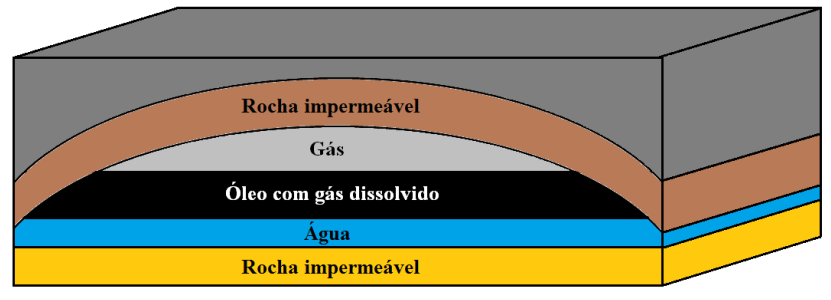

Figura 1: Ilustração de um reservatório de petróleo

produção. Neste contexto, torna-se importante o uso da simulação numérica dos fenômenos de transporte que ocorrem em meios porosos.

\subsection{Simulação numérica de reservatórios de petróleo}

Apesar da variedade de configurações, em função dos tipos de fluido e rocha encontrados na natureza, do poço perfurado e do ambiente de produção na superfície (produção em terra ou no mar), um sistema de produção de petróleo é formado por um conjunto de elementos básicos consistindo de: reservatório; poços de produção e injeção; e instalações de superfície para o recebimento dos fluidos produzidos.

Os reservatórios são formações rochosas subterrâneas, caracterizadas pelas suas porosidade e permeabilidade, que contêm fluidos retidos no seu interior devido à presença de rochas impermeáveis e/ou aquíferos (Fig. 1). Dentro de um reservatório pode-se encontrar óleo, água e/ou gás e uma distribuição não-uniforme de pressão. As fases fluidas encontram-se separadas, devido aos efeitos hidrostáticos (Guo et al.; 2007).

O conhecimento acerca do escoamento, em um reservatório de petróleo, é necessário para que se possa fazer previsões acuradas sobre a produção de hidrocarbonetos, sob diferentes condições de operação. Um projeto de recuperação pode envolver investimentos da ordem de centenas de milhões de dólares, de modo que os riscos a ele relacionados devem ser minimizados (Ertekin et al.; 2001). Alguns dos principais fatores que levam à existência de riscos e às incertezas inerentes à produção são:

- as formações rochosas heterogêneas;

- as características dos fluidos que podem variar em função da posição (devido, por exemplo, à segregação das fases);

- os mecanismos complexos de recuperação (como, por exemplo, a interação entre um reservatório e um aquífero); e

- as limitações das técnicas de previsão disponíveis para estudar os casos realísticos.

As três primeiras estão relacionadas às características do reservatório e devem ser consideradas quando da proposição dos dados de entrada para a modelagem físico-matemática de uma formação produtora. Os impactos do quarto fator podem ser reduzidos com a aplicação de técnicas da Engenharia de Reservatórios (Ertekin et al.; 2001).

As técnicas de previsão do comportamento de jazidas de hidrocarbonetos são tipicamente de três tipos: analógicas, experimentais e matemáticas.
Segundo Rosa et al. (2006), os chamados simuladores matemáticos podem ser divididos em analíticos ou numéricos. Dentre os analíticos tem-se, por exemplo, o modelo da fonte linear, a equação de balanço de materiais e as curvas de declínio. Já nos simuladores numéricos, soluções aproximadas são obtidas com o uso de ferramentas computacionais. As soluções analíticas encontram-se restritas a casos particulares, nos quais hipóteses simplificadoras são necessárias. Devido à natureza não linear das equações diferenciais parciais (EDPs), que tipicamente são usadas para descrever os escoamentos, o mais próximo possível da realidade, soluções analíticas são de difícil obtenção e, portanto, soluções aproximadas devem ser obtidas numericamente. Além das não linearidades, a presença das heterogeneidades também justifica o uso de métodos numéricos, assim como as formas geométricas irregulares dos reservatórios como, por exemplo, nas estruturas do tipo anticlinal que é o foco deste trabalho.

\subsection{Estrutura anticlinal}

Na geologia dos reservatórios de petróleo, o termo trapa (do inglês trap) diz respeito a uma configuração geométrica das formações rochosas que leva à existência de uma acumulação de petróleo, como é possível observar-se na Fig. 1. Dobras, fraturas e falhas levam à formação de barreiras internas ou externas que impedem a migração dos fluidos para outras áreas da formação subterrânea. Por exemplo, a estrutura anticlinal é definida por dois conjuntos de fraturas abertas, sendo uma paralela ao eixo anticlinal e a outra perpendicular a ele (Almahmoud et al.; 2009). Pressões laterais podem formar dobramentos que levam ao surgimento dos anticlinais, assim como a compactação de sedimentos, podendo as estruturas estarem parcialmente ou completamente preenchidas por óleo. Na trapa, com a estrutura do tipo anticlinal, tem-se uma configuração do tipo arco (Fig. 1) Esse tipo de estrutura foi primeiramente descoberta pelos geólogos no século XIX. Os anticlinais estão relacionados às trapas mais comumente encontradas no mundo (cerca de $80 \%$ do petróleo do mundo é encontrado em anticlinais) (Oser; 2017).

A complexidade da simulação numérica em estruturas anticlinais foi verificada no estudo de Eckert et al. (2014). Nesse trabalho, é discutida a dificuldade de se avaliar a diferença máxima sustentável de pressão no poro para estruturas anticlinais. Como conclusão, verificou-se que a calibração de todas as propriedades é de extrema importância para os modelos numéricos empregados na elaboração dos projetos reais, para a injeção de fluidos em estruturas do tipo anticlinal.

Uma das estratégias (Al-Mohannadi; 2004), adotada neste trabalho, para simular-se numericamente o escoamento em estruturas do tipo anticlinal é a utilização de células ativas e inativas (Behzadinasab; 2015), na qual determinadas regiões do domínio discreto são bloqueadas de forma a não existir o escoamento através delas. Assim sendo, é possível reproduzir-se uma descrição geométrica mais próxima da realidade, aproximandose o domínio de resolução da configuração em arco presente no reservatório anticlinal. 


\section{Escoamento Monofásico em Meios Porosos}

Na modelagem de escoamentos em reservatórios de petróleo, as equações governantes são EDPs tipicamente não lineares. Nestas EDPs encontram-se incorporadas as propriedades da rocha e do fluido, as características geométricas, os termos fonte e são escritas em termos da variável dependente pressão que varia no tempo e no espaço (Ertekin et al.; 2001).

\subsection{Equações governantes}

As equações governantes são obtidas considerandose as seguintes hipóteses para o modelo físicomatemático:

- o fluido é newtoniano e ligeiramente compressível;

- o meio poroso é homogêneo e anisotrópico em termos da permeabilidade;

- a porosidade é uma função linear da pressão, sendo a compressibilidade da rocha pequena e constante;

- não ocorrem efeitos eletrocinéticos, inerciais ou turbulentos;

- o escoamento é monofásico e isotérmico; e

- não há reações químicas.

\subsubsection{A equação da continuidade}

A equação da continuidade expressa o balanço de massa em uma determinada região espacial. Em reservatórios portadores de hidrocarbonetos, 0 volume de controle é uma parte do meio poroso (continuum) que contém uma ou mais fases de fluido. Para fluxos mássicos monofásicos escrevese a equação da continuidade na forma (Ertekin et al.; 2001)

$$
\frac{\partial}{\partial t}\left(\frac{\phi}{B}\right)+\nabla \cdot\left(\frac{\mathbf{v}}{B}\right)-\frac{q_{s c}}{V_{b}}=0
$$

onde $\phi=V_{p} / V_{b}$ é a porosidade, sendo $V_{p}$ o volume de espaços vazios (ou poroso) e $V_{b}=\Delta x \Delta y \Delta z$ o volume total de um bloco do reservatório (onde $\Delta x, \Delta y$ e $\Delta z$ são as arestas de um paralelepípedo que representa o reservatório), $B=\rho_{s c} / \rho$ é o fator-volume-formação (FVF), com o subscrito sc indicando as condições padrão, $\rho$ é a massa específica, $\mathbf{v}$ é a velocidade superficial do fluido (vazão de escoamento dividida pela área de seção transversal), e $q_{s c}=q_{m} / \rho_{s c}$ é a vazão definida nas condições padrão, que representa a produção ou injeção de fluido $\left(q_{m}\right)$.

\subsubsection{A lei de Darcy}

A primeira equação aplicada para descrever o movimento de fluidos em meios porosos foi obtida pelo engenheiro civil Henry Darcy, em 1856, a partir de experimentos de filtração vertical de água em colunas de areia homogênea. Em termos das propriedades do fluido, a velocidade superficial (ou aparente) do escoamento é dada pela lei clássica de Darcy (Ertekin et al.; 2001):

$$
\mathbf{v}=-\frac{\mathbf{k}}{\mu}[\nabla p-\rho g \nabla D]
$$

sendo que $\mathbf{k}$ é o tensor de permeabilidade absoluta, $\mu$ a viscosidade do fluido, $g$ a magnitude da aceleração da gravidade e $D$ é a profundidade.

\subsubsection{Compressibilidades da rocha e do fluido}

Ao longo da produção de óleo em um reservatório, a retirada de fluido do espaço poroso é acompanhada de uma variação da pressão interna da rocha, ficando ela sujeita a tensões, que podem provocar modificações nos grãos, nos poros e, algumas vezes, no volume total da rocha. Quantifica-se essa variação do volume poroso usando uma medida de compressibilidade da rocha (Rosa et al.; 2006),

$$
c_{\phi}=\frac{1}{\phi} \frac{\partial \phi}{\partial p}
$$

onde $c_{\phi}$ é o coeficiente de compressibilidade da rocha.

A porosidade varia com a pressão por causa da compressibilidade da rocha, usualmente considerada constante, sendo que esta dependência pode ser expressa, para $c_{\phi}$ pequeno e constante, na forma (Ertekin et al.; 2001):

$$
\phi=\phi^{0}\left[1+c_{\phi}\left(p-p^{0}\right)\right]
$$

onde $\phi^{0}$ e $p^{0}$ são a porosidade e a pressão de referência, respectivamente. A pressão de referência é tipicamente adotada como a pressão atmosférica ou a pressão inicial da jazida.

Para o óleo, a sua compressibilidade é caracterizada pelo coeficiente (Rosa et al.; 2006)

$$
c_{o}=\frac{1}{\rho} \frac{\partial \rho}{\partial p}
$$

\subsubsection{Equação da Difusividade Hidráulica (EDH)}

Combinando-se a lei de Darcy com a equação da conservação de massa obtém-se:

$$
V_{b} \frac{\partial}{\partial t}\left(\frac{\phi}{B}\right)=\sum_{l=x}^{z} \frac{\partial}{\partial l}\left[\frac{A_{l} k_{l}}{\mu B}\left(\frac{\partial p}{\partial l}-\rho g \frac{\partial D}{\partial l}\right)\right] \Delta l+q_{s c}
$$

onde $l=x, y, z$ e $A_{l}$ é a área da seção transversal.

Para um escoamento isotérmico, a partir da Eq. (5), para fluidos ligeiramente compressíveis e $c_{0}$ constante, é possível integrar-se essa equação usando-se o método de separação de variáveis e obter-se uma aproximação de primeira ordem dada por (Abou-Kassem et al.; 2006)

$$
\rho=\rho^{0}\left[1+c_{0}\left(p-p^{0}\right)\right]
$$

onde $\rho^{0}$ é a massa específica na pressão de referência $p^{0}$. Em seguida, reescreve-se a Eq. (7) em termos do fator-volume-formação e divide-se a mesma por $\rho_{s c}$,

$$
B=\frac{B^{0}}{\left[1+c_{o}\left(p-p^{0}\right)\right]} \text {. }
$$

A viscosidade pode ser calculada, para um fluido ligeiramente compressível e sem dependência com a 
temperatura, através da relação (Ertekin et al.; 2001)

$$
\mu=\frac{\mu^{0}}{\left[1-c_{\mu}\left(p-p^{0}\right)\right]},
$$

onde o sobrescrito " 0 " indica as condições de referência e $c_{\mu}$ representa a taxa relativa de variação da viscosidade com a pressão.

Então, a partir da definição dos coeficientes de compressibilidade da rocha e do fluido pode-se reescrever o termo transiente, da Eq. (6), na forma

$$
\frac{\partial}{\partial t}\left(\frac{\phi}{B}\right)=\left(\frac{\phi c_{o}}{B^{0}}+\frac{c_{\phi} \phi^{0}}{B}\right) \frac{\partial p}{\partial t}=\Gamma \frac{\partial p}{\partial t},
$$

de modo que a EDH é escrita, finalmente, na forma

$$
V_{b} \Gamma \frac{\partial p}{\partial t}=\sum_{l=x}^{z} \frac{\partial}{\partial l}\left[\frac{A_{l} k_{l}}{\mu B}\left(\frac{\partial p}{\partial l}-\rho g \frac{\partial D}{\partial l}\right)\right] \Delta l+q_{s c}
$$

onde $l=x, y, z$ e o coeficiente $\Gamma$ incorpora os efeitos de compressibilidade da rocha e do fluido.

\subsubsection{Condições inicial, de contorno e o termo fonte}

Para resolver-se a Eq. (11), condições inicial e de contorno apropriadas devem ser fornecidas. Como condição inicial utiliza-se

$$
p(x, y, z, t=0)=p_{\text {ini }}(x, y, z)
$$

onde $p_{\text {ini }}$ é a pressão inicial antes do reservatório ser influenciado pela produção/injeção de fluidos.

Como condições de contorno externas (limites externos do reservatório), pressão ou vazão prescrita podem ser utilizadas. Para um reservatório selado no formato de um paralelepípedo, de arestas $L_{x}, L_{y}$ e $L_{z}$, considera-se aqui que o gradiente de pressão é nulo nas fronteiras do reservatório:

$$
\left(\frac{\partial p}{\partial x}\right)_{x=0, L_{x}}=0, \quad\left(\frac{\partial p}{\partial y}\right)_{y=0, L_{y}}=0, \quad\left(\frac{\partial p}{\partial z}\right)_{z=0, L_{z}}=0 \text {. }
$$

Os poços são considerados como sendo fronteiras internas e, no caso de uso de coordenadas cartesianas, o termo fonte está diretamente relacionado ao acoplamento poço-reservatório. Para a representação dos poços utiliza-se uma expressão que relaciona a pressão no poço, $p_{w f}$ (se a vazão é especificada), ou a taxa de produção, $q_{s c}$ (se a pressão no poço é especificada), com a pressão $p$ do fluido no meio poroso. Na simulação numérica de reservatórios, quando pretende-se determinar a pressão nos poços, em simulações em geometria empregando coordenadas cartesianas, tipicamente usa-se

$$
q_{s c}=-J_{w}\left(p-p_{w f}\right)
$$

com o Índice de Produtividade sendo definido como $J_{w} \equiv G_{w} /(\mu B)$, onde $G_{w}$ representa o fator geométrico do poço, função das propriedades do fluido, da geometria, da rocha e do tipo de modelagem de poço aplicada (Ertekin et al.; 2001).
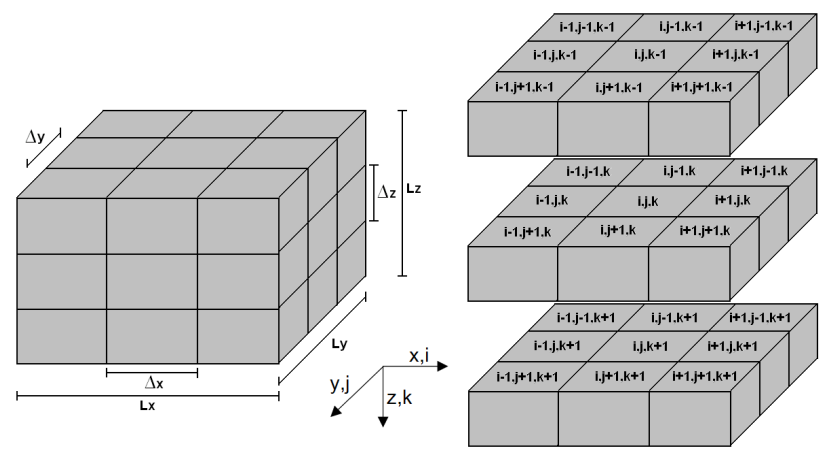

Figura 2: Discretização de uma reservatório em coordenadas $x y z$

\section{Metodologia Numérica}

Como a EDH (Eq. (11)) é não linear ela não pode, no caso geral, ser resolvida analiticamente e, portanto, utiliza-se na sua resolução numérica o Método das Diferenças Finitas (MDF), que é o mais utilizado na indústria petrolífera (Ertekin et al.; 2001).

\subsection{Discretização}

Neste trabalho, utiliza-se uma discretização baseada em blocos centrados (Ertekin et al.; 2001). Na Fig. 2 mostra-se um reservatório retangular discretizado por $n_{x} \times n_{y} \times n_{z}$ blocos, onde para fim ilustrativo tomou-se $n_{x}=n_{y}=n_{z}=3$. Em geral, as dimensões dos blocos não precisam ser iguais, de forma que

$$
\sum_{i=1}^{n_{x}} \Delta x_{i}=L x, \quad \sum_{j=1}^{n_{y}} \Delta y_{i}=L y, \quad \sum_{k=1}^{n_{z}} \Delta z_{i}=L_{z}
$$

onde os índices $i, j$ e $k$ referem-se ao centro dos blocos nas direções $x, y$ e $z$, respectivamente. As fronteiras dos blocos são indicadas através da soma da fração $\pm 1 / 2$ a um dos índices $i, j$ e $k$, em função da fronteira que se deseja representar. Por exemplo, a fronteira entre as células $i, j, k$ e $i+1, j, k$ é representada por $i+1 / 2, j, k$.

Conforme pode ser constatado, a Eq. (11) contém derivadas espaciais e temporal. Assim sendo, elas precisam ser substituídas por aproximações do tipo diferenças. Em se tratando da malha da Fig. 2 e de uma formulação totalmente implícita, o primeiro termo contendo as derivadas espaciais da Eq. (11) pode ser discretizado por um esquema do tipo diferença finita centrada:

$$
\begin{aligned}
{\left[\frac{\partial}{\partial x}\left(T_{x} \frac{\partial p}{\partial x}\right) \Delta x\right]_{i, j, k}^{n+1} } & \approx \frac{1}{\Delta x_{i, j, k}}\left\{\left[\left(T_{x} \frac{\partial p}{\partial x}\right) \Delta x\right]_{i+1 / 2, j, k}^{n+1}\right. \\
& \left.-\left[\left(T_{x} \frac{\partial p}{\partial x}\right) \Delta x\right]_{i-1 / 2, j, k}^{n+1}\right\}, \quad(16)
\end{aligned}
$$

onde desconsiderou-se os efeitos gravitacionais. $\mathrm{O}$ sobrescrito $n+1$ indica o tempo no qual as pressões são desconhecidas, $\Delta x_{i, j, k}$ é o espaçamento da célula $i$ na direção $x$. Formas semelhantes são obtidas para as direções y e $z$, considerando-se que $\Delta y_{i, j, k}$ e $\Delta z_{i, j, k}$ 
são os espaçamentos da malha nas direções $y$ e $z$, respectivamente. Os coeficientes $T_{x}, T_{y}$ e $T_{z}$ nessas aproximações são as transmissibilidades do meio poroso (Ertekin et al.; 2001), dadas por

$$
\mathrm{T}_{l_{i \pm \frac{1}{2}, j, k}^{n+1}}=\left(\frac{A_{l} k_{l}}{\mu B \Delta l}\right)_{i \pm \frac{1}{2}, j, k}^{n+1}, \quad l=x, y, z .
$$

Novamente, optando-se por aproximações do tipo diferenças centradas,

$$
\left(\frac{\partial p}{\partial x}\right)_{i+\frac{1}{2}, j, k}^{n+1} \approx \frac{p_{i+1, j, k}^{n+1}-p_{i, j, k}^{n+1}}{x_{i+1, j, k}-x_{i, j, k}}=\frac{p_{i+1, j, k}^{n+1}-p_{i, j, k}^{n+1}}{\triangle x_{i+\frac{1}{2}, j, k}}
$$

$$
\left(\frac{\partial p}{\partial x}\right)_{i-\frac{1}{2}, j, k}^{n+1} \approx \frac{p_{i, j, k}^{n+1}-p_{i-1, j, k}^{n+1}}{x_{i, j, k}-x_{i-1, j, k}}=\frac{p_{i, j, k}^{n+1}-p_{i-1, j, k}^{n+1}}{\triangle x_{i-\frac{1}{2}, j, k}}
$$

com formas análogas correspondentes para as direções $y$ e $z$.

No termo de acúmulo é aplicada uma expansão conservativa, a fim de se preservar o balanço de massa nos níveis de tempo sucessivos ( $n$ e $n+1$ ) (Ertekin et al.; 2001),

$$
\Gamma_{i, j, k}^{n+1}=\frac{V_{b, i, j, k}}{\Delta t}\left(\frac{\phi^{o} c_{\phi}}{B^{n+1}}+\frac{\phi^{n} c_{o}}{B^{0}}\right)_{i, j, k}
$$

e a derivada temporal é substituída por uma aproximação do tipo diferença finita atrasada

$$
\left(\frac{\partial p}{\partial t}\right)_{i, j, k}^{n+1} \approx \frac{p_{i, j, k}^{n+1}-p_{i, j, k}^{n}}{\Delta t} .
$$

Considerando-se o uso de uma solução por uma iteração de Picard (Nick et al.; 2013), assume-se que os valores são conhecidos nos níveis $v$ enquanto que os valores a serem determinados o serão nos níveis $v+1$. Assim, a forma final discretizada linearizada da EDH é

$$
\begin{array}{r}
\Gamma_{i, j, k}^{n+1, v}\left(p_{i, j, k}^{n+1, v+1}-p_{i, j, k}^{n}\right)=T_{x_{i+\frac{1}{2}, j, k}^{n+1, v}}\left(p_{i+1, j, k}^{n+1, v+1}-p_{i, j, k}^{n+1, v+1}\right) \\
+T_{x_{i-\frac{1}{2}, j, k}^{n+1, v}}^{n+1}\left(p_{i-1, j, k}^{n+1, v+1}-p_{i, j, k}^{n+1, v+1}\right)+T_{y_{i, j+\frac{1}{2}, k}^{n+1, v}}^{n+1}\left(p_{i, j+1, k}^{n+1, v+1}-p_{i, j, k}^{n+1, v+1}\right) \\
+T_{y_{i, j-\frac{1}{2}, k}^{n+1, v}}\left(p_{i, j-1, k}^{n+1, v+1}-p_{i, j, k}^{n+1, v+1}\right)+T_{z_{i, j, k+\frac{1}{2}}^{n+1, v}}^{n+1}\left(p_{i, j, k+1}^{n+1, v+1}-p_{i, j, k}^{n+1, v+1}\right) \\
+T_{z_{i, j, k-\frac{1}{2}}}^{n+1, v}\left(p_{i, j, k-1}^{n+1, v+1}-p_{i, j, k}^{n+1, v+1}\right)-\left(q_{s c}\right)_{i, j, k}^{n+1, v+1} . \quad(22)
\end{array}
$$

\subsection{Acoplamento poço-reservatório}

O tratamento do poço, injetor ou produtor, em simulação de reservatórios introduz certas dificuldades na simulação em função, entre outras, da discrepância de escalas existente entre o tamanho do poço e o do reservatório, bem como da geometria de poços complexos. Exceto para simulações que fazem uso de coordenadas cilíndricas, o uso de termos fonte/sorvedouro são necessários para a implementação das condições de contorno envolvendo os poços (Ertekin et al.;
2001). Por conseguinte, técnicas de acoplamento poço-reservatório foram desenvolvidas com a finalidade de relacionar as pressões nas células do reservatório com a pressão no poço, pelo uso, por exemplo, de equações envolvendo termos de fonte/sorvedouro (Souza; 2013).

Considera-se, aqui, uma expressão para a vazão no poço escrita em termos do índice de produtividade $\left(J_{w}\right)$ para um poço vertical,

$$
q_{s c}=-\underbrace{\frac{2 \pi \sqrt{k_{x} k_{y}} \Delta z}{B \mu \ln \left(r_{e q} / r_{w}\right)}}_{=J_{w}}\left(p_{i, j, k}-p_{w f, k}\right)
$$

onde $r_{e q}$ é o raio equivalente (Peaceman; 1983),

$$
r_{e q}=0,28 \frac{\sqrt{\sqrt{k_{y} / k_{x}}(\Delta x)^{2}+\sqrt{k_{x} / k_{y}}(\Delta y)^{2}}}{\sqrt[4]{k_{y} / k_{x}}+\sqrt[4]{k_{x} / k_{y}}} .
$$

A vazão de produção total prescrita é representada por $Q_{s c}$, obtida a partir do somatório de todas as vazões $q_{s c}$ das células pelas quais passa o poço vertical.

\subsection{Regiões inativas}

Os reservatórios portadores de hidrocarbonetos possuem formas irregulares, resultado dos processos geológicos atuantes ao longo de milhões de anos. Para permitir um melhor ajuste da malha computacional à geometria irregular, quando utiliza-se uma malha estruturada, os simuladores comerciais costumam fornecer a opção de se escolher quais células são consideradas inativas para fins de escoamento (Ertekin et al.; 2001). Nesse tipo de estratégia, como pode ser visto na Fig. 3, algumas das células do reservatório são tornadas inativas, ou seja, bloqueadas ao escoamento. Isto pode ser feito, por exemplo, atribuindo o valor zero para a permeabilidade dessas células. Dessa forma, utilizando-se uma média harmônica para o cálculo da transmissibilidade, as conexões entre essas células são tornadas nulas. Uma outra alternativa é a de se anular diretamente as transmissibilidades relacionadas às células inativas.

De modo similar ao presente trabalho, o simulador de Behzadinasab (2015) também permite a definição de regiões inativas e faz uso do processamento em paralelo, empregando o Message Passing Interface (MPI) (Grama et al.; 2003). Contudo, ressalta-se que a versão serial desse simulador, no momento do cálculo das propriedades que dependem da pressão do óleo (como por exemplo, a viscosidade), computa as alterações para todas as células da malha (ativas e inativas). Já na versão paralelizada, a determinação dessas propriedades é realizada exclusivamente nas células ativas. Baseando-se nessa ideia, quando das modificações introduzidas na versão preexistente do simulador monofásico (Werneck et al.; 2016), além de um rastreador para definir a geometria do reservatório anticlinal, também foram codificados rastreadores para que as propriedades que dependem da pressão sejam apenas calculadas para as células ativas. As transmissibilidades só são identicamente 


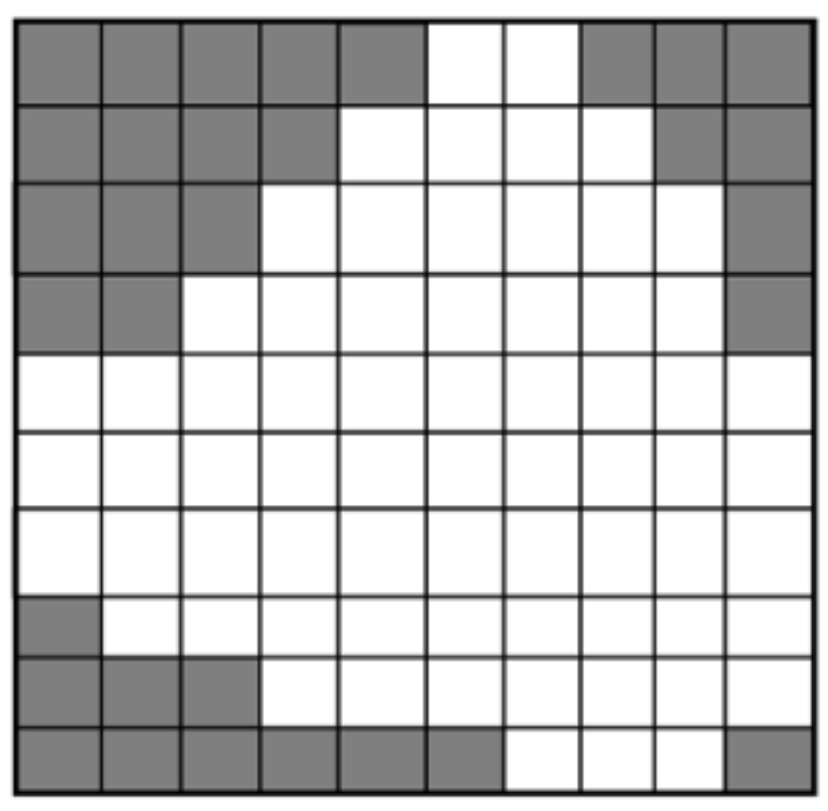

Figura 3: Regiões inativas (cinza) e ativas (branco) em um modelo bidimensional

determinadas nas faces que conectam duas células ativas.

A definição das regiões inativas que anulam as regiões de fronteira pode levar a diferentes versões do simulador, impactando na resolução numérica do sistema de equações algébricas. Por exemplo, é possível anular-se as permeabilidades das regiões inativas, computar as propriedades para todas as células e calcular-se todas as transmissibilidades durante toda a simulação. Uma segunda opção, adotada neste trabalho, consiste em anular-se as permeabilidades das regiões inativas, determinarse as propriedades exclusivamente para as células ativas e só atualizar-se as transmissibilidades na interface das células ativas. Nessas duas estratégias, as pressões nas células ativas e inativas são determinadas quando da solução do sistema de equações. Entretanto, as pressões das células inativas permanecem com o valor estimado quando do início da simulação. Uma terceira alternativa, otimizada e também encontrada na literatura (Abou-Kassem and Ertekin; 1992), obtém a solução do sistema de equações apenas para as células ativas. Por fim, uma quarta opção seria a construção de uma malha nãoestruturada, considerando apenas as regiões ativas, e dispensando a fase de anulação das permeabilidades.

As três primeiras alternativas são empregadas conjuntamente com malhas estruturadas. A primeira é a mais simples de ser implementada e a menos eficiente em termos computacionais. Embora um pouco mais elaborada, a segunda também pode ser introduzida sem maiores dificuldades e apresenta uma melhor eficiência computacional. A terceira diferencia-se da segunda no que diz respeito à matriz dos coeficientes do sistema algébrico, como considera somente as células ativas demandará um menor esforço computacional. A última implica no uso de um gerador de malhas não-estruturadas. Entretanto, a geometria do reservatório anticlinal pode ser representada com uma maior precisão. O principal esforço computacional adicional estará associado à geração da malha não-estruturada. Todas as alternativas apresentadas podem resultar na obtenção de resultados acurados mediante o emprego de malhas suficientemente refinadas.

Para reproduzir-se uma geometria que seja a mais próxima da estrutura do tipo anticlinal e implementar-se o rastreador, que busca as regiões inativas e anula as permeabilidades das células inativas, faz-se uma adaptação do esquema de interpolação que pode ser encontrado em Ertekin et al. (2001), o que implica no uso de malhas que possuam valores uniformes de $\Delta x, \Delta y$ e $\Delta z$. Tal esquema tem sido adotado em simuladores de reservatórios para a interpolação das propriedades físicas dependentes da pressão, tais como a viscosidade, a fim de reduzir o custo computacional. Assim sendo, ao invés do emprego de correlações para a determinação dessas propriedades em cada célula, em todas as iterações e em todos os passos de tempo, os valores interpolados são obtidos diretamente de uma tabela.

Portanto, a estratégia sugerida (Ertekin et al.; 2001) implica na busca de valores em uma tabela, cujas colunas são a propriedade a ser interpolada e a pressão, considerando que a diferença entre as pressões

$$
\Delta p=p_{m+1}-p_{m}
$$

é constante, sendo $p_{1}, p_{2}, \ldots, p_{n}$ os valores de pressão para os quais conhece-se valor da propriedade desejada, existindo $m=1,2, \ldots, n$ linhas na tabela. Então, para um dado valor $p^{*}$, para o qual deseja-se saber o valor interpolado, faze-se

$$
m=1+I\left(\frac{p^{*}}{\Delta p}\right)
$$

onde a função I retorna o valor inteiro do resultado da divisão. Dessa forma, determina-se que a propriedade desejada deve ser interpolada usando-se os valores de $p^{*}$ e $p_{m}$.

$\mathrm{Na}$ busca pelas regiões inativas e a consequente anulação das permeabilidades das células que as formam, a Eq. (26) foi adaptada de modo a se fazer a busca pelos índices que determinam as regiões inativas na malha computacional original. Elas são rastreadas dentro do paralelepípedo formado por todas as células (ativas e inativas). Por exemplo, se o reservatório tem dimensões $L_{x}, L_{y}$ e $L_{z}$ e a região determinada por $\left(L_{x 1}, L_{x 2}\right),\left(L_{y 1}, L_{y 2}\right)$ e $\left(L_{z 1}, L_{z 2}\right)$ é inativa, realizam-se as buscas

$$
m l_{1}=1+I\left(\frac{L_{l 1}}{\Delta l}\right), \quad m l_{2}=1+I\left(\frac{L_{l 2}}{\Delta l}\right),
$$

onde $l=x, y, z$, de maneira que dentre os valores de 0 a $n_{x}$ para $x, 0$ a $n_{y}$ para $y$ e 0 a $n_{z}$ para $z$, as células que se encontram entre $m x_{1}$ e $m x_{2}, m y_{1}$ e $m y_{2}$ e $m z_{1}$ e $m z_{2}$ receberão um valor nulo para a permeabilidade. Nessa busca inicial, também se coletam informações para que, durante a simulação, apenas sejam calculadas as propriedades nas células ativas e, exclusivamente, se atualize as transmissibilidades nas faces localizadas entre células ativas. O rastreamento permite, por exemplo, a representação de um caso como o apresentado na Fig. 4. 


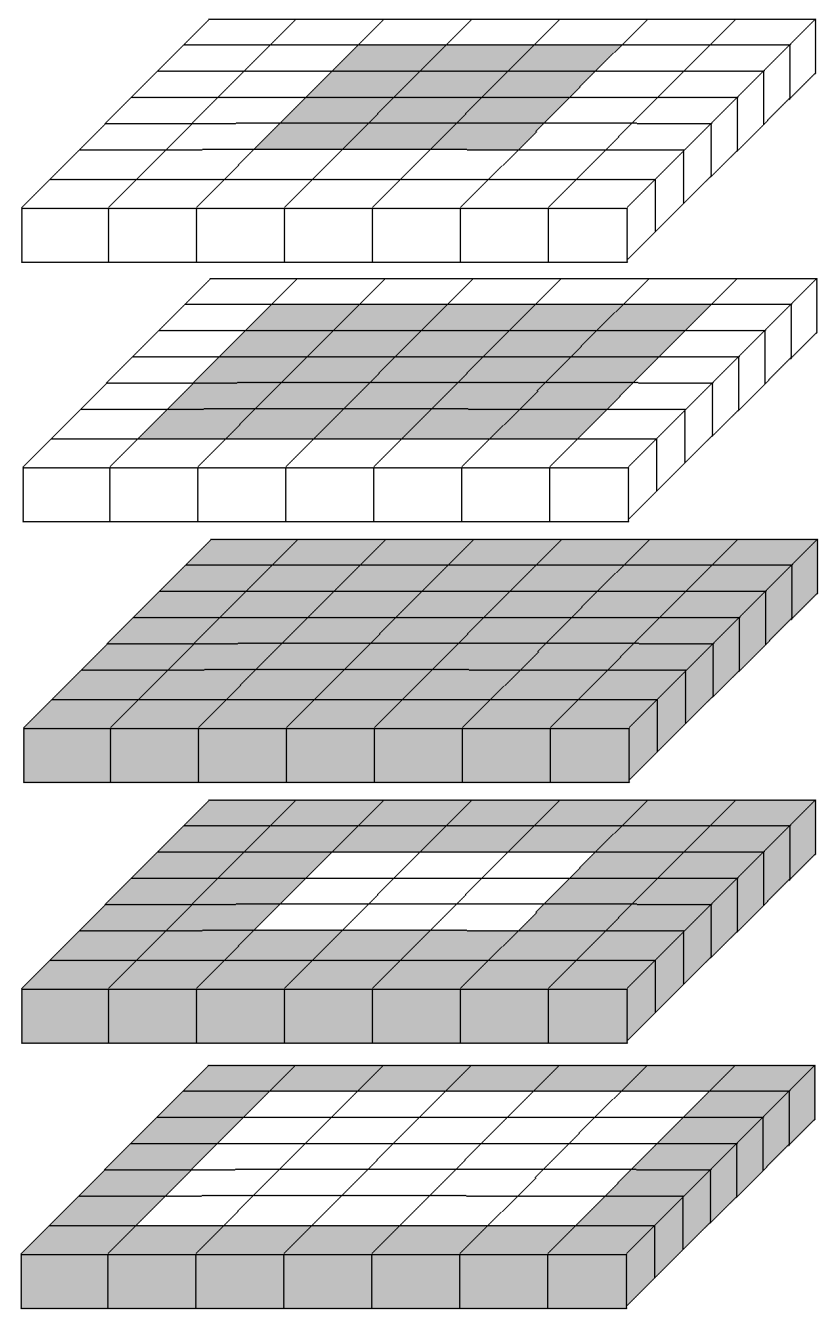

Figura 4: Reservatório anticlinal de 5 camadas: células ativas (cinza) e inativas (branca)

\subsection{Solução do sistema de equações algébricas}

O Algoritmo 1 descreve as etapas de resolução numérica do escoamento monofásico de óleo (ligeiramente compressível) em um reservatório de petróleo formado por rochas ligeiramente compressíveis. Nele, $F_{\Delta t}$ representa a razão de crescimento do passo de tempo (linha 4), cujo valor limite é $\Delta t_{f}$ (passo de tempo final). Nas linhas 12 a 14 é prevista a resolução do sistema algébrico via o método dos Gradientes Conjugados.

\subsubsection{Técnicas de compressão de matrizes}

O objetivo final de toda simulação numérica é o de alcançar resultados que sejam confiáveis e próximos dos seus correspondentes reais (físicos). Além desse desafio, também depara-se, a qualquer tempo, com as barreiras impostas pelas limitações do hardware dos computadores como, por exemplo, a velocidade dos processadores e a capacidade de armazenamento.

Em se tratando da capacidade de memória, algumas técnicas de compressão de matrizes vêm sendo desenvolvidas e destaca-se (Antunes Filho and Xavier; 2015):

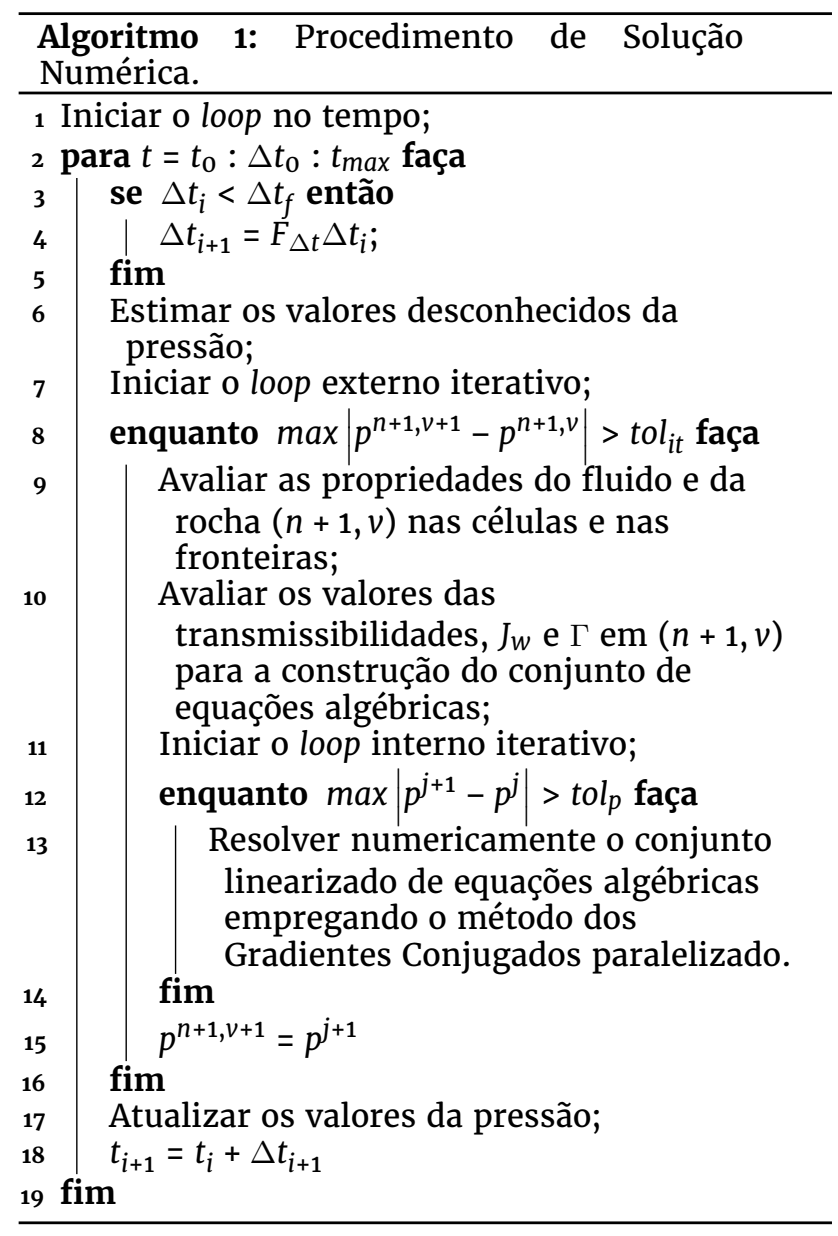

- Matriz no Formato Denso ou Dense Format;

- Matriz no Formato Coordenado ou Coordinate Format (COO);

- Matriz no Formato de Linha Comprimida ou Compressed Sparse Row (CSR);

- Matriz no Formato de Coluna Comprimida ou Compressed Sparse Column (CSC); e

- Matrix Market Format.

A técnica Compressed Sparse Row (CSR) ou Linha Esparsa Comprimida é uma forma de armazenamento de matrizes esparsas que utiliza três vetores para referenciar e armazenar os valores não nulos de uma matriz esparsa (Werneck et al.; 2016) e foi a escolhida para ser aqui utilizada. Essencialmente, o vetor matrixValues armazena os valores não nulos da matriz, o vetor mtxIndj armazena os índices referentes às colunas em que os elementos não nulos estão posicionados na matriz e o vetor nonzerosInRow armazena os índices identificando a posição no vetor matrixValues onde se inicia uma nova linha da matriz. Caso a matriz seja simétrica, será necessário armazenar apenas os valores da diagonal principal e dos elementos acima (ou abaixo) da mesma.

$\mathrm{O}$ tamanho dos vetores varia dinamicamente em função do número de elementos nulos na matriz dos coeficientes, que depende diretamente do número de células ativas e inativas presentes na malha computacional. Eles são alocadas integralmente na memória RAM durante a execução das simulações. 


\subsubsection{OpenMP}

Quando depara-se com simulações numéricas que envolvem a resolução de grandes sistemas algébricos, parte substancial do custo computacional é dedicado à sua resolução numérica através de algum método (normalmente) iterativo. Logo, a fim de minimizar o tempo execução, pode-se fazer uso da computação de alto desempenho. Dentre as várias técnicas disponíveis encontra-se o processamento em paralelo (distribuído e/ou multiprocessado) (Venâncio Júnior and Silva; 2004).

As técnicas de processamento paralelo mais difundidas atualmente são as de memória distribuída ou compartilhada. A primeira se aplica a máquinas com vários processadores que possuem seu próprio recurso de memória e são interconectadas através de uma rede local. Atualmente, o padrão MPI (Message Passing Interface) tem sido o mais utilizado nesses casos e o seu funcionamento se dá basicamente através de troca de dados entre os processadores. Por outro lado, as técnicas de memória compartilhada são utilizadas em ambientes que possuem vários núcleos de processamento compartilhando a mesma memória. Uma ferramenta que vem sendo bastante empregada na implementação dessa técnica é o OpenMP (Open Multi-Processing), tendo sido desenvolvido especificamente de modo a facilitar as aplicações com memória compartilhada (Werneck; 2016).

Em algumas situações, o uso do OpenMp é mais indicado do que o MPI, embora os dois possam ser utilizados em geral. O OpenMP usa um modelo de memória compartilhada que, geralmente, é apropriado somente em computadores onde os processadores acessam uma memória compartilhada. Já o MPI é mais indicado para o seu uso em clusters, onde a memória (e os processadores) são distribuídos e a memória compartilhada não é um recurso central da arquitetura. Entretanto, no caso de se ter um código serial já existente, como era o do presente trabalho, e que precisa ser melhorado no que diz respeito ao tempo total de execução quando da resolução de problemas práticos de interesse, a sua paralelização representa, provavelmente, a situação mais comum e difícil de ser executada. Portanto, neste cenário, o paralelismo incremental é muito mais viável com o OpenMP uma vez que com o MPI seria, presumivelmente, necessário uma reescritura de parte considerável do código numérico.

O OpenMP é uma interface de programação (Application Programming Interface) (API), baseada no modelo de programação paralela de memória compartilhada para arquiteturas de múltiplos processadores (Chapman et al.; 2008) e é composto de três componentes básicos: Diretivas de Compilação; Biblioteca de Execução; e Variáveis de Ambiente, e pode ser usado com os compiladores Fortran, C e $\mathrm{C}++$ em ambientes Unix e Windows (Sistemas Multithreads) (Brunschen and Brorsson; 2000).

O OpenMP representa um padrão que define como os compiladores devem gerar códigos paralelos através da incorporação de diretivas que indicam como as tarefas serão divididas entre os processadores ou cores. Os programas escritos para o uso do OpenMP utilizam um modelo de execução conhecido como fork-join (Werneck; 2016), esquematizado na Fig. 5. As unidades de execução processarão as partes do código computacional que

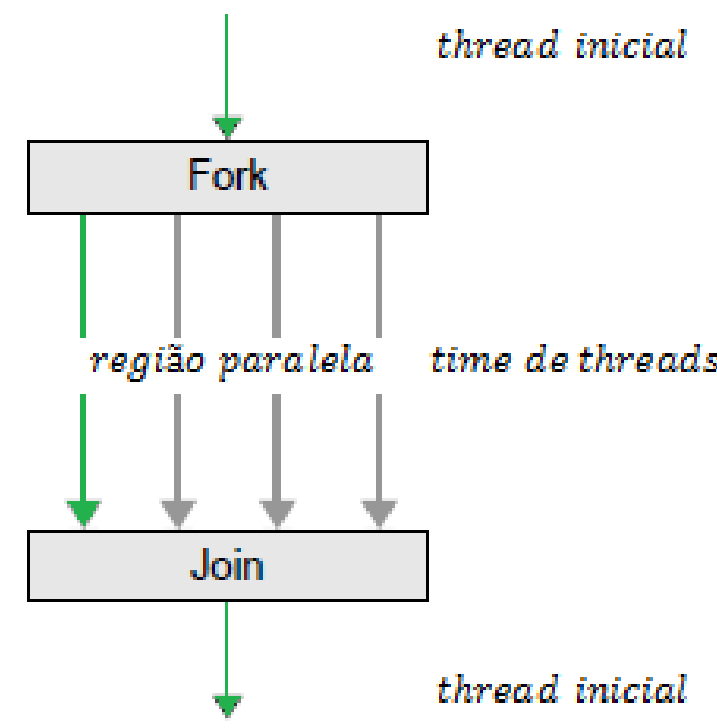

Figura 5: Modelo de programação OpenMP

são definidas pelas diretivas OpenMP (ARB; 2011) (de forma implícita ou explícita).

Um programa com diretivas OpenMP inicia com uma única thread ou thread inicial (ou mestre), que executa sequencialmente as instruções até encontrar a diretiva parallel (Hemstad; 2013), que indica o processamento em paralelo. Ela é responsável por designar as regiões do código que serão processadas por várias threads (regiões paralelas). Cada thread possui sua própria pilha de execução e compartilha o mesmo endereço de memória com as demais threads do mesmo processo, sendo que cada processo possui um acesso de memória próprio.

Quando uma thread encontra a diretiva parallel é criado um grupo de threads (fork) (Torelli and Bruno; 2004). Assim, agora, cada thread será responsável pela execução das instruções paralelizadas. Concluída a execução em paralelo o grupo de threads sincronizase em uma barreira ${ }^{1}$ implícita com a thread mestre. Neste instante, o grupo de threads conclui a sua execução e a thread mestre continua o processamento sequencial (join) até que uma nova região paralela seja identificada ou até que o programa chegue ao final da sua execução (Chapman et al.; 2008). Se um grupo de threads encarregado do processamento em paralelo de uma região encontrar outra diretiva parallel, cada thread desse grupo dará origem a um novo grupo de threads e a mesma se tornará também uma thread mestre (Werneck; 2016).

A programação multithread com OpenMP possui várias vantagens que estimulam a sua adoção. As principais são (Chapman et al.; 2008):

- normalmente são feitas poucas alterações no código serial existente (facilidade de conversão);

- possui uma robusta estrutura de suporte à programação paralela;

- fácil compreensão do uso das diretivas;

- suporte ao paralelismo aninhado; e

${ }^{1}$ Implica no fato de que nenhuma thread pode prosseguir até que todas as demais threads do grupo atinjam esse ponto no programa (Chapman et al.; 2008). 
- possibilita o ajuste dinâmico do número de threads.

\subsubsection{O Método dos Gradientes Conjugados}

O Método dos Gradientes Conjugados (Hestenes and Stiefel; 1952) é utilizado neste trabalho na resolução iterativa do sistema linearizado de equações algébricas. No caso de matrizes de coeficientes esparsas de grande porte, os métodos iterativos permitem a otimização da alocação de memória e uma resolução eficiente (Canal; 2000). Em geral, o método converge somente após um grande número de iterações e nem sempre a sua convergência é assegurada. Por convergência do método entende-se a obtenção da solução do sistema algébrico, após um número finito de iterações, para uma dada tolerância de variação entre as soluções numéricas obtidas sequencialmente.

Na sua essência, o método dos gradientes conjugados consiste na execução de passos, a cada iteração, na direção oposta a do campo vetorial dado pelo gradiente da função objetivo, sem repetir a direção anterior, até encontrar o mínimo estrito e global dessa função. A minimização ocorre nos chamados espaços de Krylov, gerados a partir do resíduo de cada iteração (Canal; 2000). As aproximações $x_{k+1}$ e os resíduos $r_{k+1}$ são atualizados em cada iteração utilizando o escalar $\alpha_{k}$ e as direções de busca $p_{k+1}$ são atualizadas utilizando o escalar $\beta_{k}$ (vide o Algoritmo 2).

Como pode ser observado no algoritmo, que introduz a versão paralelizada do método usando a interface OpenMP (Werneck; 2016), a aplicação consiste na realização de várias operações entre vetores e matrizes como, por exemplo, a soma e o produto interno de vetores e a multiplicação de uma matriz por um vetor. Como essas operações são responsáveis diretamente pela maior parte do esforço computacional exigido, devido à sua natureza complexa (recursiva) do ponto de vista computacional, elas foram prioritariamente paralelizadas.

Quando do uso das cláusulas schedule para a divisão das iterações de um laço, do código numérico, pelas threads três possibilidades são possíveis: static, dynamic e guided. Optou-se, neste trabalho, por empregar a primeira opção (default) onde as iterações são agrupadas em conjuntos estaticamente atribuídos às threads.

\section{Resultados}

A Tab. 1 contém os dados de entrada para um caso padrão de forma que, a menos quando especificado em contrário, esses são os valores utilizados nas simulações. Utilizou-se, na execução do código numérico, uma servidora Dell PowerEdge T620 com 2 processadores (12 núcleos) Intel Xeon E5-2620 (CPU de 2,00 GHz) com um total de 24 threads, $16 \mathrm{~Gb}$ de memória RAM; o sistema operacional Linux OpenSUSE Leap 42.1 (64 bits), Kernel 4.1.36-44default; e o compilador gcc versão 4.8.5 (SUSE Linux). Os resultados são apresentados para a pressão do meio poroso ao longo do eixo $x$, para $y=L_{y} / 2 \mathrm{e} z=L_{z} / 2$, para o tempo máximo de produção estipulado.

Os testes foram implementados tomando como referência um reservatório anticlinal de cinco

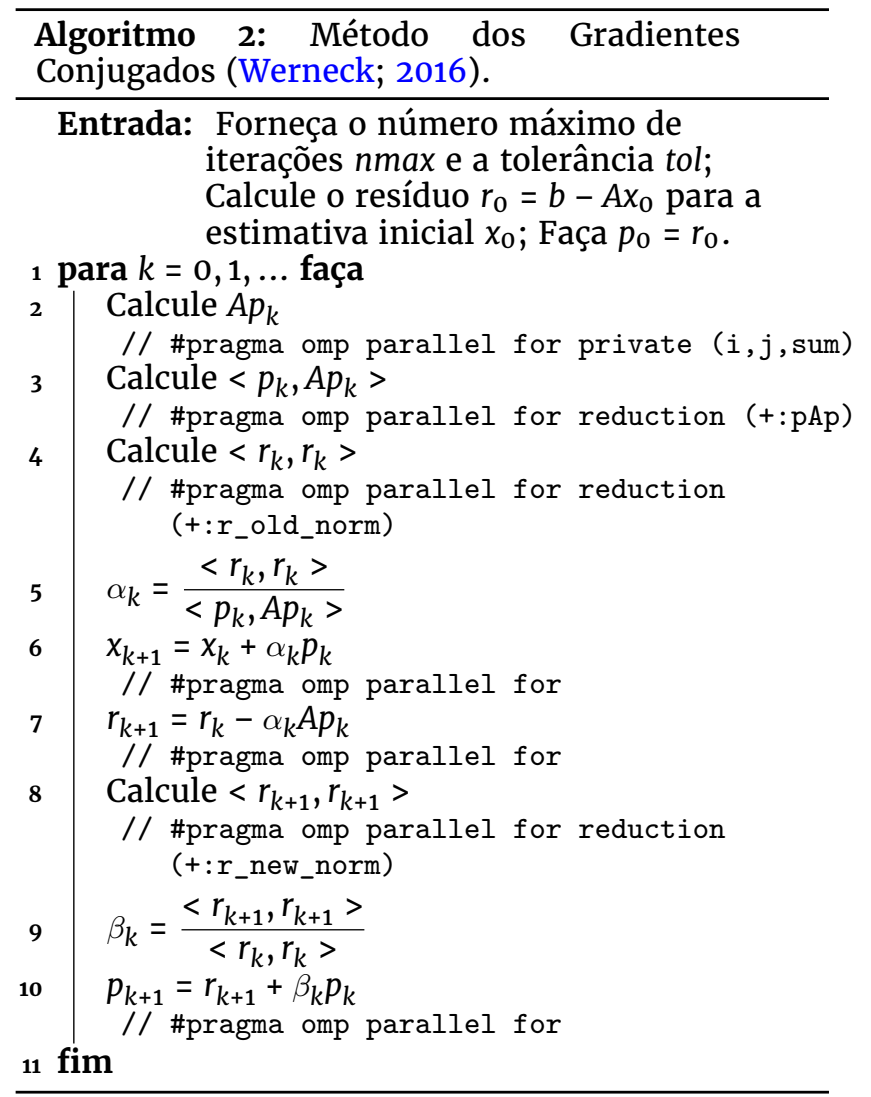

camadas na direção $z$, enumeradas de cima para baixo. Nas Camadas 1 e 2 foram tornadas inativas as regiões nas fronteiras em $x$ e $y$, enquanto que nas Camadas 4 e 5 as regiões centrais no plano $x y$ foram tidas como inativas. A Camada 3 permaneceu completamente ativa. A Fig. 4 fornece, de forma esquematizada, a representação gráfica do reservatório aqui descrito. $\mathrm{Na}$ apresentação dos resultados, o reservatório anticlinal de cinco camadas é definido como sendo do Tipo 1 , o anticlinal com três camadas é nomeado como anticlinal do Tipo 2 e os anticlinais que não são simétricos são identificados pelas nomenclaturas Tipo 1 e Tipo 2 assimétricos. Esses reservatórios são construídos, respectivamente, ao se tornar inativas as regiões no plano $x y$ dos reservatórios simétricos de Tipos 1 e 2, que ocorre na camada central em relação ao eixo $z$, a partir de um de seus vértices no plano $x y$. As malhas consideradas no estudo do refinamento espacial são introduzidas na Tab. 2.

A Fig. 6 traz os resultados obtidos com o estudo do refinamento de malha. Na sequência das Malhas 1 até 3 os resultados apontam para evidências de convergência numérica. Para um método numérico consistente e estável, à medida que fazemos os incrementos espaciais tenderem a zero a solução numérica deve tender para a única solução da equação diferencial parcial que está sendo resolvida, em se tratando de um problema bem-posto. Na comparação entre os $n$ valores da pressão $\left(p_{i}\right)$ determinados com as três primeiras malhas, com relação aos valores calculados com a malha mais refinada $\left(p_{4}\right)$, empregou-se o desvio médio quadrático normalizado pela pressão média $\left(p_{m}\right): D N_{i}=\frac{\sqrt{\sum_{i=1}^{n}\left[p_{i}(x)-p_{4}(x)\right]^{2}}}{p_{m}}$, para $i=1,2,3$. Para $o$ conjunto de dados 
Tabela 1: Caso padrão para as simulações

\begin{tabular}{lcc}
\hline Parâmetro & Valor & Unidade \\
\hline$n_{x}$ & 97 & - \\
$n_{y}$ & 97 & - \\
$n_{z}$ & 51 & - \\
tol $l_{i t}$ & $1 \times 10^{-6}$ & psi \\
tol & $1 \times 10^{-3}$ & psi \\
$F_{\Delta t}$ & 1,5 & - \\
$t_{\text {max }}$ & 1.000 & dia \\
$\Delta t_{0}$ & 1.0 & dia \\
$\Delta t_{f}$ & 20 & dia \\
$Q_{s c} \sum q_{s c}$ & -100 & STB/dia \\
$k_{x}$ & $5 \times 10^{-3}$ & Darcy \\
$k_{y}$ & $5 \times 10^{-3}$ & Darcy \\
$k_{z}$ & $2 \times 10^{-3}$ & Darcy \\
$\phi$ & 0,2 & adimensional \\
$\phi^{0}$ & 0,2 & adimensional \\
$c_{\phi}$ & $5 \times 10^{-5}$ & psi ${ }^{-1}$ \\
$p^{0}$ & 6.000 & psi \\
$p_{\text {ini }}$ & 6.000 & $\mathrm{psi}$ \\
$\rho^{0}$ & 52,4 & $\mathrm{~kg} / \mathrm{m}^{3}$ \\
$\mu$ & 1,0 & $\mathrm{cp}$ \\
$c_{o}$ & $1 \times 10^{-4}$ & $\mathrm{psi}{ }^{-1}$ \\
$B$ & 1,25 & $\mathrm{RB} / \mathrm{STB}$ \\
$L_{x}$ & $6.370,0$ & $\mathrm{ft}$ \\
$L_{y}$ & $6.370,0$ & $\mathrm{ft}$ \\
$L_{z}$ & 135,0 & $\mathrm{ft}$ \\
$L_{p}$ & 81,0 & $\mathrm{ft}$ \\
$N_{\text {threads }}$ & 12 & - \\
\hline & & \\
& &
\end{tabular}

Tabela 2: Malhas espaciais

\begin{tabular}{ccccc}
\hline Células & Malha 1 & Malha 2 & Malha 3 & Malha 4 \\
\hline$n_{x}$ & 25 & 49 & 97 & 193 \\
$n_{y}$ & 25 & 49 & 97 & 193 \\
$n_{z}$ & 15 & 27 & 51 & 99 \\
$n_{x} \times n_{y} \times n_{z}$ & 9.375 & 64.828 & 479.859 & 3.687 .651 \\
\hline
\end{tabular}

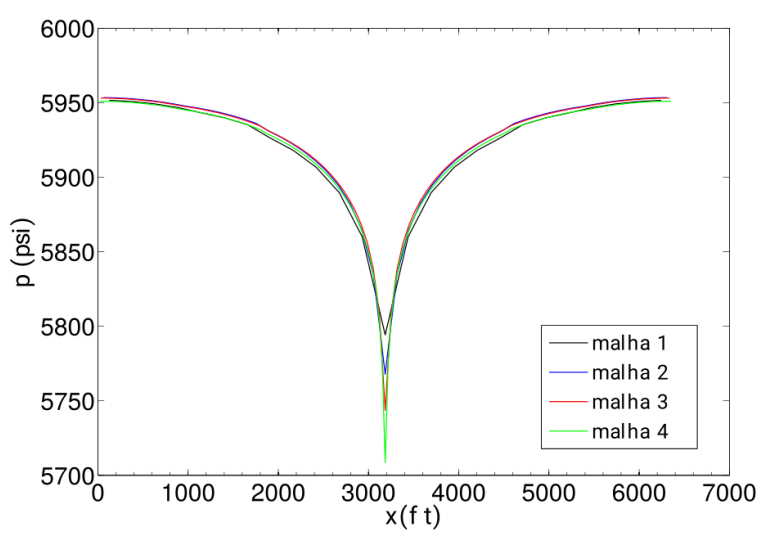

Figura 6: Refinamento espacial da malha

testados, entende-se que todas as malhas já se encontram suficientemente refinadas e apresentaram os seguintes valores para o desvio médio quadrático normalizado: $D N_{1}=0,0191, D N_{2}=0,0152$ e $D N_{3}=0,0115$, respectivamente para as Malhas 1, 2 e 3. Para se ter uma ideia, na simulação de reservatórios, na escala de campo, muitas vezes as células possuem milhares de ft de comprimento nas direções $x$ e $y$. Portanto, para os demais testes realizados optou-se pelo uso da Malha 3.

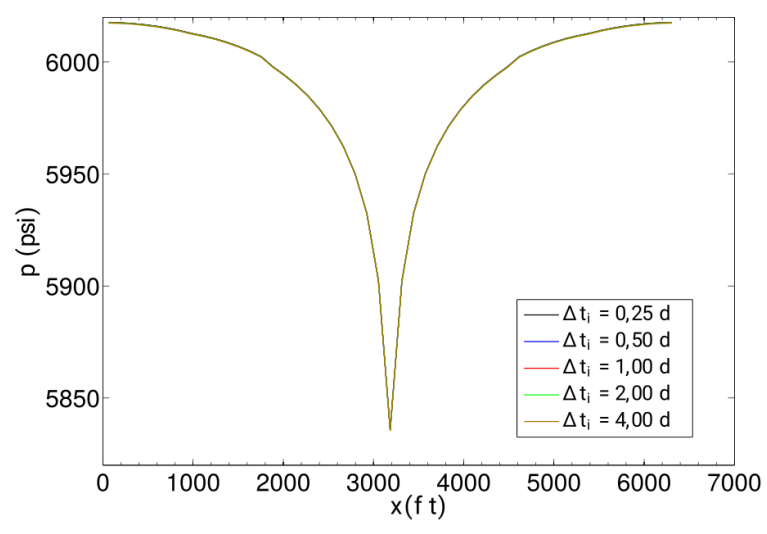

Figura 7: Refinamento do passo de tempo $(p)$

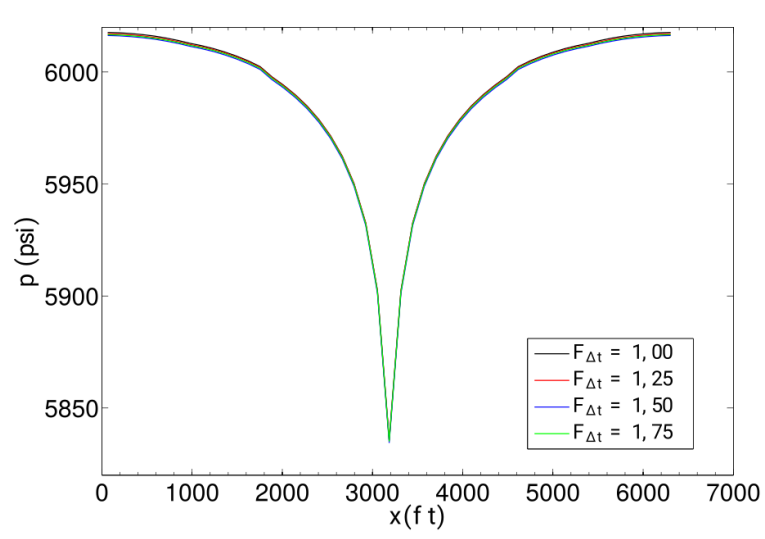

Figura 8: Variação da razão de crescimento do passo de tempo

Estudos de refinamento também foram realizados variando-se o passo de tempo. A Fig. 7 mostra os resultados para a pressão em função da variação do passo de tempo. Na Fig. 8 pode-se verificar a convergência à medida que varia-se os fatores multiplicativos dos incrementos de tempo. $O$ passo de tempo inicial é representado por $\Delta t_{i}$ e a sua razão de crescimento por $F_{\Delta t}$. Por outro lado, a Fig. 9 representa uma ampliação de parte da Fig. 8, para fins de visualização. Em virtude dos resultados obtidos, pode-se verificar que não ocorreram diferenças significativas ao se variar os parâmetros, ficando as maiores diferenças em torno de 1 psi (considerandose $t \geq 4$ dias). Nesses testes simulou-se 200 dias de produção. Nas demais simulações utilizou-se, portanto, um passo de tempo inicial de 1 dia e uma razão de crescimento igual a 1,5, com o passo de tempo parando de crescer quando alcançados 20 dias. O tempo total de simulação adotado foi de 1.000 dias de produção.

\subsection{Análise de Desempenho}

O estudo do desempenho computacional foi realizado variando-se as malhas computacionais e o número de threads ( $\left.N_{\text {threads }}\right)$ empregadas nas simulações. Os resultados são apresentados em termos dos tempos computacionais e do valor do speedup, calculado 


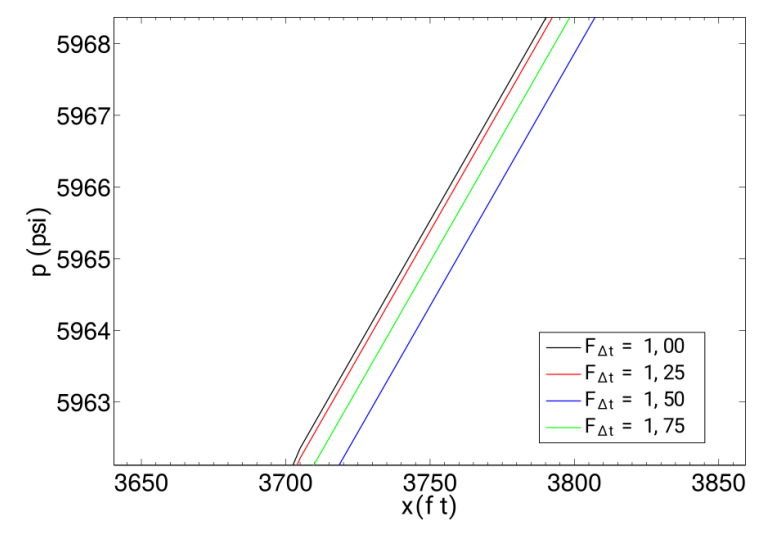

Figura 9: Variação da razão de crescimento do passo de tempo (ampliação)

dividindo-se o tempo de simulação obtido com 1 thread pelo tempo correspondente com $n$ threads. Os tempos e os valores do speedup encontram-se tabelados para as diferentes malhas, sendo que os valores do speedup também são mostrados na forma de gráficos para cada malha.

Na Tab. 3 pode-se notar que para a Malha 1 a simulação foi finalizada rapidamente, de modo que uma comparação do desempenho computacional é difícil de ser feita (muitas simulações com o mesmo tempo de execução). Contudo, pode-se verificar que houve um ganho evidente no speedup em função da utilização de mais do que uma thread. A Fig. 10 mostra um gráfico do speedup em função do número de threads. Percebe-se a existência de uma região de crescimento do ganho em eficiência computacional, seguido de uma de estabilização a partir do uso de 6 threads. Essa estabilização do ganho é interrompida por uma queda de desempenho com o uso de 20 threads.

Tabela 3: Desempenho: Malhas 1 e 2

\begin{tabular}{ccccc}
\hline & \multicolumn{2}{c}{ Malha 1 } & \multicolumn{2}{c}{ Malha 2} \\
$N_{\text {threads }}$ & Tempo (s) & speedup & Tempo (s) & speedup \\
\hline 1 & 8 & 1,00 & 109 & 1,00 \\
2 & 5 & 1,60 & 60 & 1,81 \\
4 & 4 & 2,00 & 37 & 2,95 \\
6 & 3 & 2,67 & 28 & 3,89 \\
8 & 3 & 2,67 & 24 & 4,54 \\
10 & 3 & 2,67 & 21 & 5,19 \\
12 & 3 & 2,67 & 23 & 4,74 \\
14 & 3 & 2,67 & 25 & 4,36 \\
16 & 3 & 2,67 & 24 & 4,54 \\
18 & 3 & 2,67 & 22 & 4,96 \\
20 & 4 & 2,00 & 21 & 5,19 \\
22 & 3 & 2,67 & 21 & 5,19 \\
24 & 3 & 2,67 & 21 & 5,19 \\
\hline
\end{tabular}

A Tab. 3 e a Fig. 10 também trazem os resultados para a Malha 2. Nota-se, no comparativo com os resultados obtidos com a Malha 1, que os tempos computacionais cresceram como resultado do maior número de células usados nas simulações. O valor do speedup também cresceu, alcançando um pico de 5,19 para 10, 20, 22 e 24 threads. A Fig. 10 mostra que tem-se um crescimento do ganho em eficiência

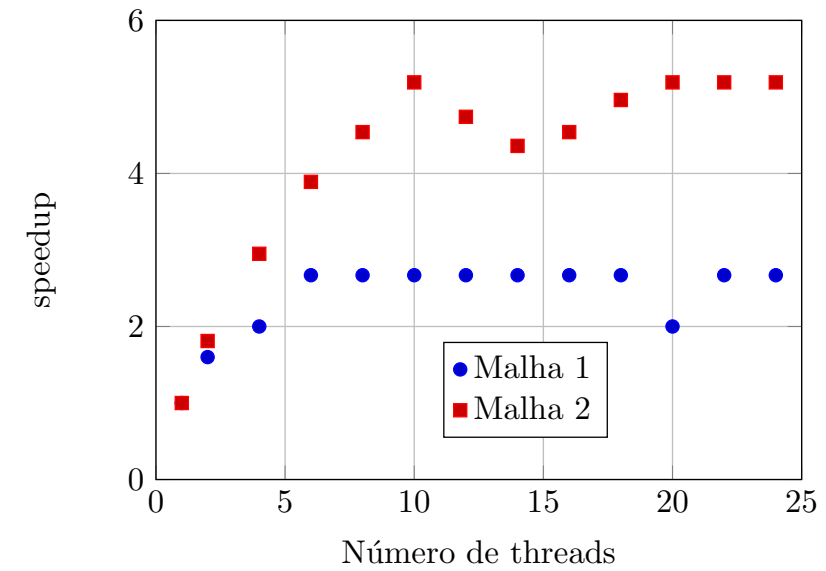

Figura 10: speedup para as Malhas 1 e 2

computacional com o aumento no número de threads, até 10 threads. A partir desse número, não há mais um ganho significativo e, inclusive, ocorre uma queda de desempenho a partir de 12 e até 18 threads. Entretanto, novamente, os tempos de simulação estão muito próximos a partir do uso de 8 threads até o número máximo de 24.

Em se tratando da Malha 3, os resultados já trazem uma escala de tempo de execução que permite uma melhor análise do comportamento da eficiência computacional, como consequência do uso de uma maior quantidade de threads. A Tab. 4 e a Fig. 11 mostram os respectivos resultados determinados com o uso dessa malha. Conforme esperado, verificase que os tempos de execução cresceram quando comparados com os das Malhas 1 e 2. Também ampliou o valor do speedup, chegando a um máximo de 6,25 para 12 threads. Assim como nos casos anteriores, a Fig. 11 apresenta os valores do speedup em função do número de threads e nota-se um crescimento do speedup com o aumento no número de threads, até 12 threads, sendo que para a faixa posterior não há mais ganho e, novamente, existe uma queda no desempenho para valores acima de 14 threads.

Tabela 4: Desempenho: Malhas 3 e 4

\begin{tabular}{ccccc}
\hline & \multicolumn{2}{c}{ Malha 3 } & \multicolumn{2}{c}{ Malha 4} \\
$N_{\text {threads }}$ & Tempo (s) & speedup & Tempo (s) & speedup \\
\hline 1 & 1.106 & 1,00 & 12.709 & 1,00 \\
2 & 602 & 1,84 & 6.815 & 1,87 \\
4 & 349 & 3,17 & 4.381 & 2,90 \\
6 & 264 & 4,19 & 2.979 & 4,27 \\
8 & 218 & 5,07 & 2.422 & 5,25 \\
10 & 190 & 5,82 & 2.195 & 5,79 \\
12 & 177 & 6,25 & 1.972 & 6,44 \\
14 & 229 & 4,83 & 2.472 & 5,14 \\
16 & 211 & 5,24 & 2.380 & 5,33 \\
18 & 198 & 5,59 & 2.229 & 5,70 \\
20 & 190 & 5,82 & 2.111 & 6,02 \\
22 & 181 & 6,11 & 2.012 & 6,31 \\
24 & 178 & 6,21 & 1.917 & 6,63 \\
\hline
\end{tabular}

Os melhores resultados em termos de speedup foram obtidos para a Malha 4 , cujos resultados encontram-se na Tab. 4 e na Fig. 11. Com ela foram obtidos os tempos computacionais mais altos, 


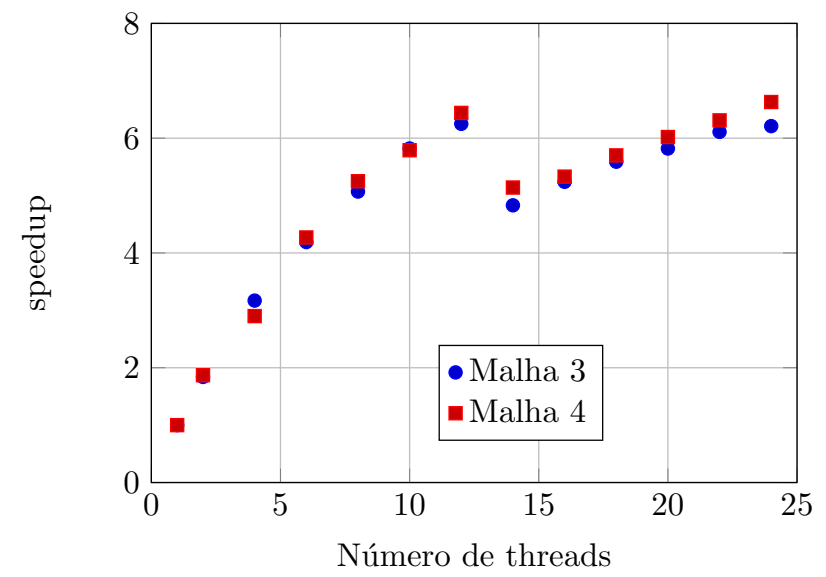

Figura 11: speedup para as Malhas 3 e 4

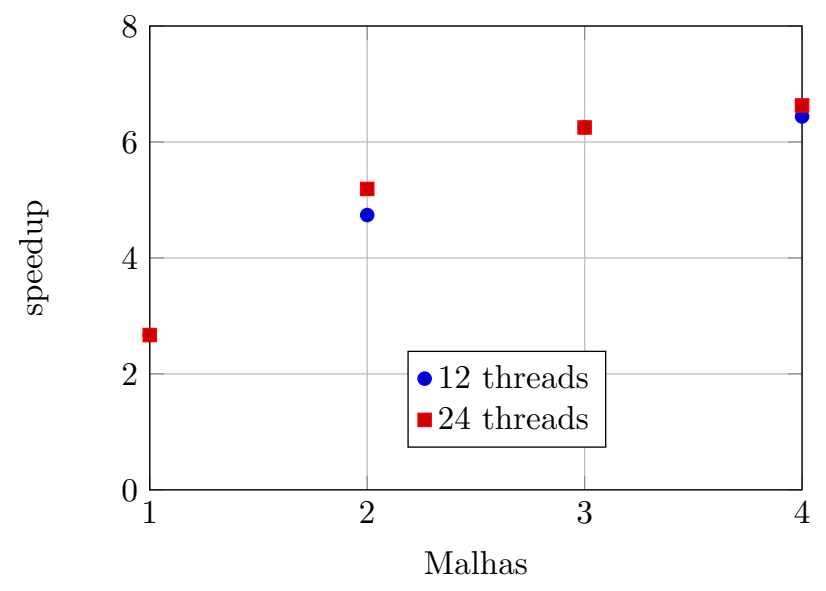

Figura 12: speedup para 12 e 24 threads

o que é justificado em função do maior número de incógnitas no sistema de equações a ser resolvido. 0 speedup chegou a um pico de 6,63 para 24 threads. Da visualização da Fig. 11 observa-se o padrão de crescimento do desempenho e o aparecimento da região de estagnação, com uma queda no desempenho mais significativa, novamente, a partir de 14 threads.

Para auxiliar na análise do speedup, a Fig. 12 traz os resultados obtidos com a variação das malhas empregando-se, respectivamente, 12 e 24 threads. Constata-se que o ganho em eficiência computacional tende a se estabilizar com o aumento no número de células. Além disso, as curvas possuem um comportamento similar tanto para 12 como para 24 threads, mesmo após ter sido duplicado o seu o número.

As explicações para a ausência de um ganho em eficiência computacional em função do aumento do número de threads utilizadas está associada à existência de barreiras internas, conforme mencionado em Chapman et al. (2008). Assim sendo, para que seja possível continuar numa trajetória de ganho para um número crescente de threads, uma programação do código numérico mais específica e elaborada em termos do uso do OpenMP seria necessária, mas esta (embora importante) não foi a finalidade inicial deste trabalho.

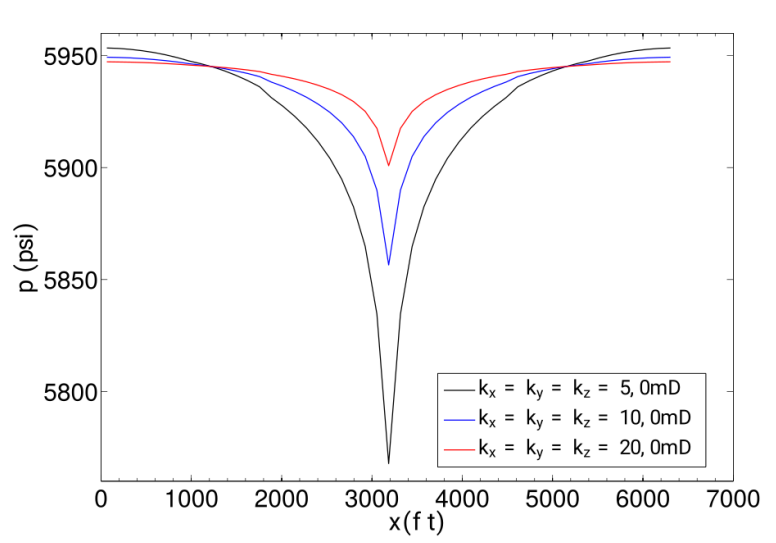

(a) Isotrópico.

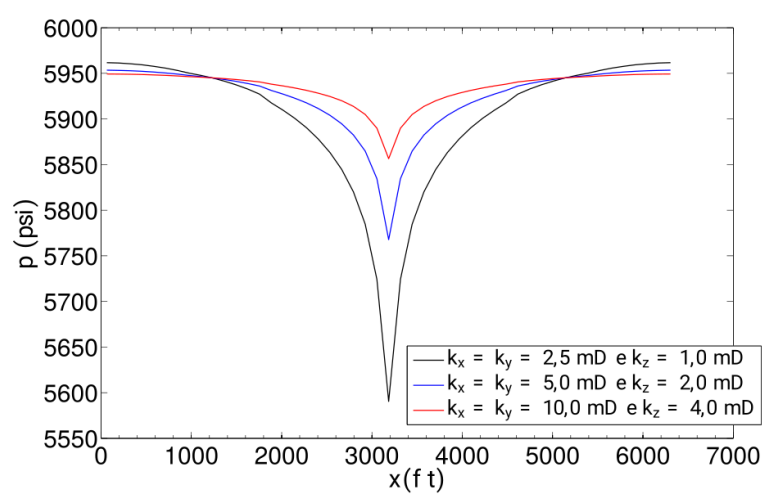

(b) Anisotrópico.

Figura 13: Pressão do reservatório: diferentes permeabilidades

\subsection{Análise de Sensibilidade}

Uma outra etapa deste estudo diz respeito aos testes de sensibilidade dos resultados numéricos, em função da variação de alguns dos parâmetros físicos que são essenciais na simulação de reservatórios. Os gráficos elaborados mostram a variação da pressão em função das propriedades modificadas da rocha e do fluido, além da sua dependência com a geometria do reservatório. Compara-se, por exemplo, situações nas quais o reservatório é irregular ou regular, sempre tomando por base o reservatório anticlinal denominado de Tipo 1 (simétrico).

As Figs. 13(a), 14(a), 13(b) e 14(b) apresentam os resultados para a pressão do reservatório e do poço em função da variação da permeabilidade para um meio isotrópico e anisotrópico, respectivamente. Da Fig. 13(a) observa-se que, de forma consistente com o esperado da física do escoamento, as menores pressões aparecem na região do poço para o menor valor da permeabilidade, devido à maior dificuldade do fluido escoar através do meio poroso em uma região de baixa permeabilidade. Neste caso, a maior queda de pressão ocorre quando deseja-se manter a mesma vazão de escoamento para as diferentes permeabilidades consideradas nos testes.

Por outro lado, nas fronteiras externas do reservatório (por exemplo, em $\chi=0$ ) as maiores pressões são observadas para os menores valores 


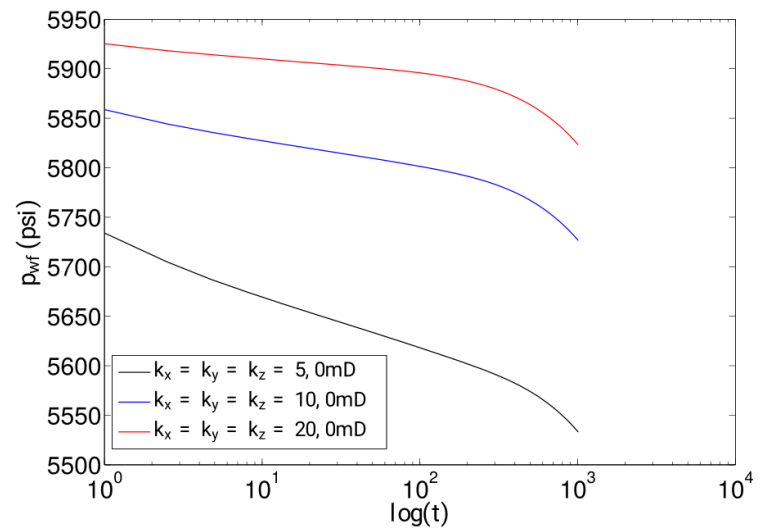

(a) Isotrópico.

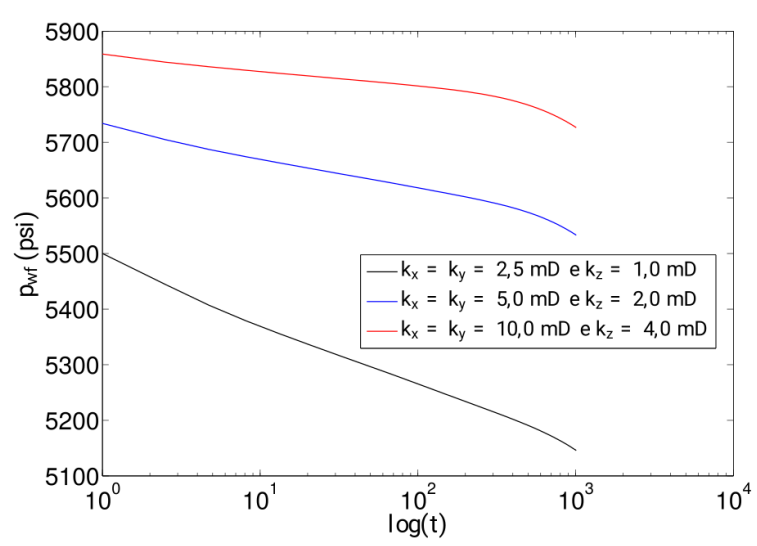

(b) Anisotrópico.

Figura 14: Pressão do poço: diferentes permeabilidades

da permeabilidade. Isso ocorre uma vez que para os valores mais baixos da permeabilidade mais tempo transcorre para que o efeito da mudança de pressão seja propagado pelo meio poroso. Na Fig. 14(a) verifica-se que não foi constatado o aparecimento do artefato numérico (introduz um comportamento do tipo estocagem, que não existe no problema simulado) comumente mencionado na literatura (Souza; 2013), quando se usa a técnica de acoplamento poço-reservatório de Peaceman, para os tempos de produção aqui simulados. Novamente, para o menor valor da permeabilidade observa-se a maior queda de pressão, e uma maior demora na percepção dos efeitos de fronteira (fim da região linear dos gráficos de $\left.p_{w f} \times \log t\right)$. Conclusões semelhantes são obtidas com relação ao reservatório anisotrópico, Figs. 13(b) e 14(b), sendo que para os sistemas com a maior permeabilidade equivalente (raiz cúbica do produto das permeabilidades nas três direções espaciais) a pressão decai menos tanto no poço quanto no reservatório (Bourdet; 2002).

As Figs. 15 e 16 mostram os resultados para as pressões variando-se a compressibilidade do óleo $c_{0}$. De forma condizente com o que é previsto pela física do escoamento em meios porosos, para os menores valores da compressibilidade os efeitos de fronteira ocorrem mais rápido e a queda de pressão é maior (Bourdet; 2002). Quanto mais

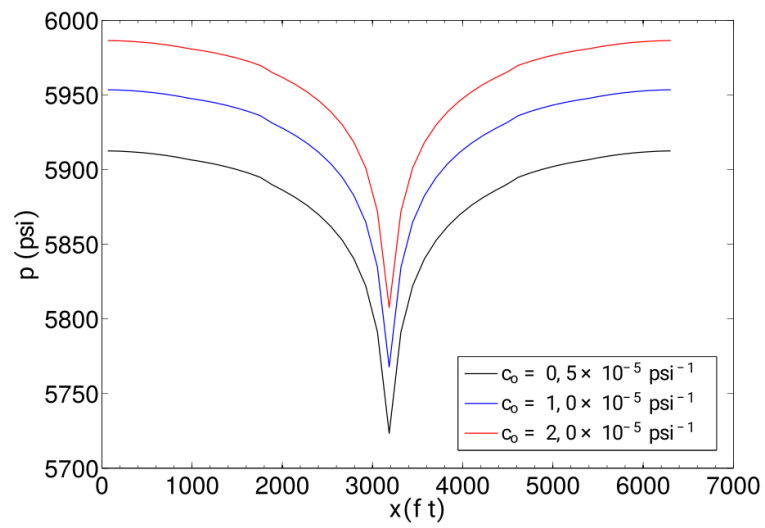

Figura 15: Pressão do reservatório: diferentes coeficientes de compressibilidade $c_{0}$

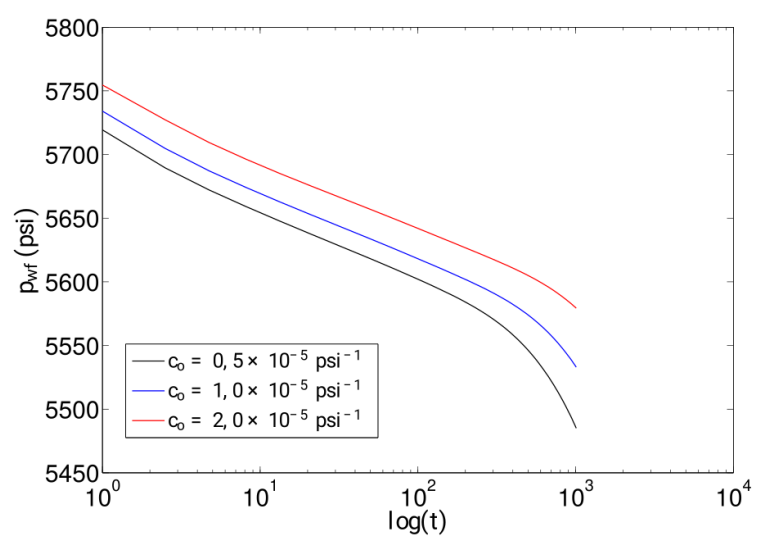

Figura 16: Pressão do poço: diferentes coeficientes de compressibilidade $c_{0}$

compressível for o fluido, tendo-se em vista que as demais características das simulações são mantidas inalteradas, mais baixo será o gradiente de pressão para que o escoamento ocorra para uma vazão previamente fixada, e mais tempo decorrerá até que os efeitos de fronteira sejam percebidos no reservatório. Mais uma vez, não foi constatado o aparecimento do artefato numérico quando da determinação de $p_{w f}$. Destaca-se que a técnica de acoplamento poço-reservatório não foi desenvolvida para o caso de reservatórios anticlinais (Peaceman; 1983). Também, em relação à reprodução adequada dos efeitos físicos, os resultados da Fig. 17 confirmam o fato de que quanto maior a vazão de produção, maior a queda de pressão no reservatório (Rosa et al.; 2006).

Na Fig. 18 pode-se notar que a queda de pressão do poço no reservatório anticlinal é maior do que a do reservatório regular (ativo), que serve de base para a criação das regiões inativas e da configuração do reservatório anticlinal, além de possuir um valor menor da pressão no meio poroso quando comparada à do reservatório regular. Isso se deve a dois fatores, o primeiro está relacionado ao menor comprimento aberto $\left(L_{p}\right)$ ao escoamento para o poço em relação ao caso do anticlinal: $81 \mathrm{~m}$ no anticlinal contra $135 \mathrm{~m}$ no reservatório regular (a maior queda de pressão ocorre para que a mesma vazão seja mantida quando 


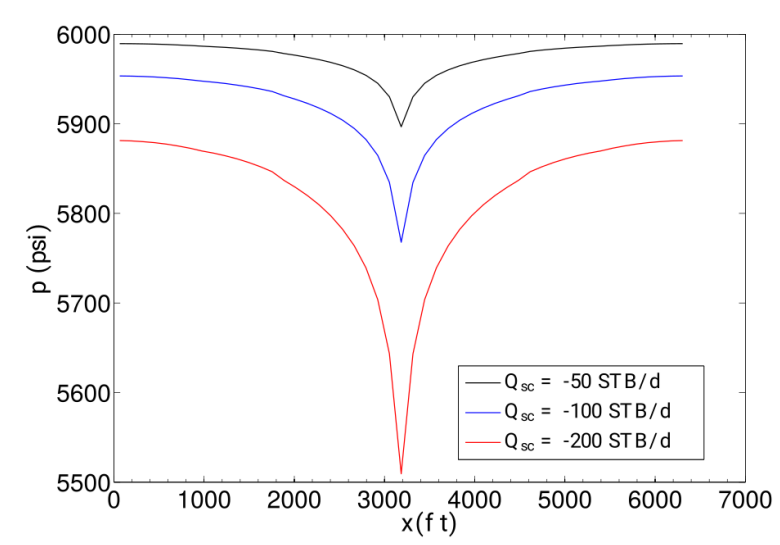

Figura 17: Pressão do reservatório: diferentes vazões de produção $Q_{s c}$

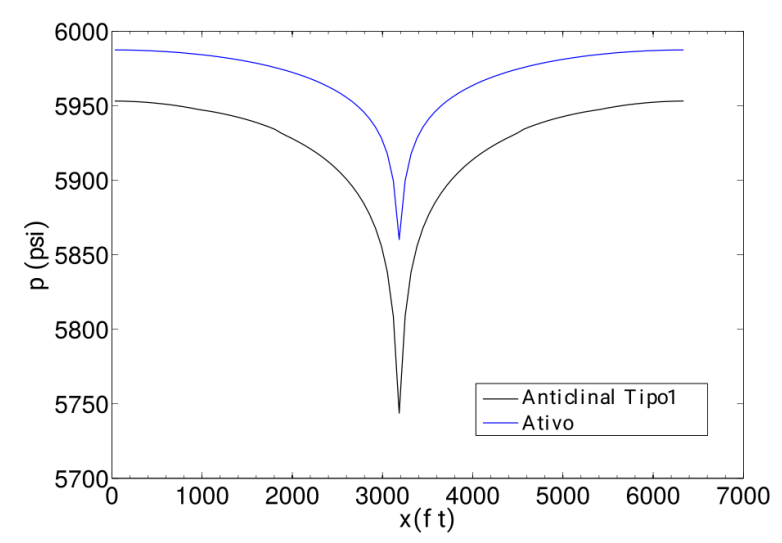

Figura 18: Pressão do reservatório: reservatórios anticlinal do Tipo 1 e regular (Ativo)

o comprimento aberto ao fluxo do reservatório para o poço é menor). O segundo fator está relacionado ao menor volume de fluido disponível para a produção na estrutura anticlinal considerada, que foi construída a partir do reservatório regular mediante a introdução de células inativas. Portanto, há menos fluido no reservatório anticlinal em comparação à quantidade existente no reservatório regular.

Vê-se, na Fig. 19, uma comparação entre os resultados obtidos para a pressão dos reservatórios anticlinal de 3 camadas (Tipo 2) e de 5 camadas (Tipo 1) na direção $z$. No anticlinal com 3 camadas pode-se perceber uma maior queda de pressão quando $x=1.820 \mathrm{ft}$ e $x=4.550 \mathrm{ft}$, locais que correspondem às mudanças de camadas. No anticlinal com 5 camadas essa alteração é menos aparente (mais gradual), contudo ela também encontra-se presente. No que diz respeito ao poço produtor, também existem entre esses dois reservatórios diferenças entre os comprimentos abertos ao fluxo mássico (como no caso do anticlinal com 5 camadas e o regular): no anticlinal de 3 camadas o poço tem $90 \mathrm{~m}$ enquanto que no anticlinal de 5 camadas ele possui $81 \mathrm{~m}$. Assim, como resultado do menor número de regiões inativas e do menor comprimento de poço, a pressão decai mais para o reservatório de 5 camadas.

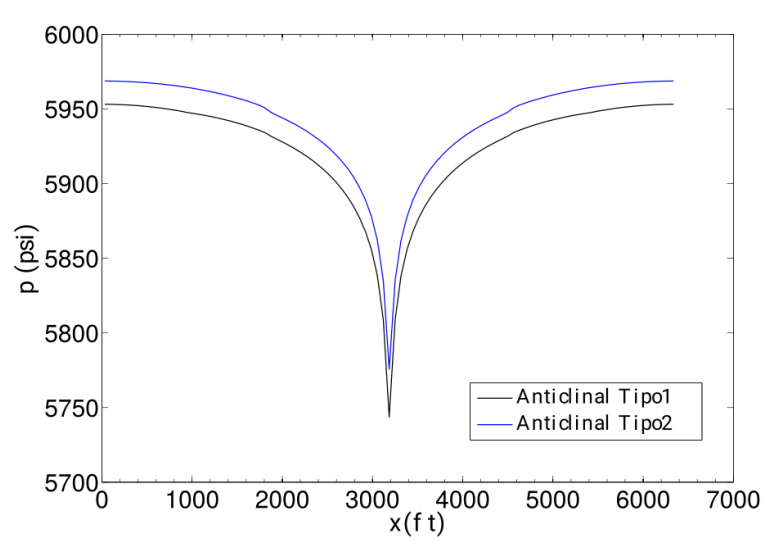

Figura 19: Pressão do reservatório: reservatórios anticlinais de Tipos 1 e 2

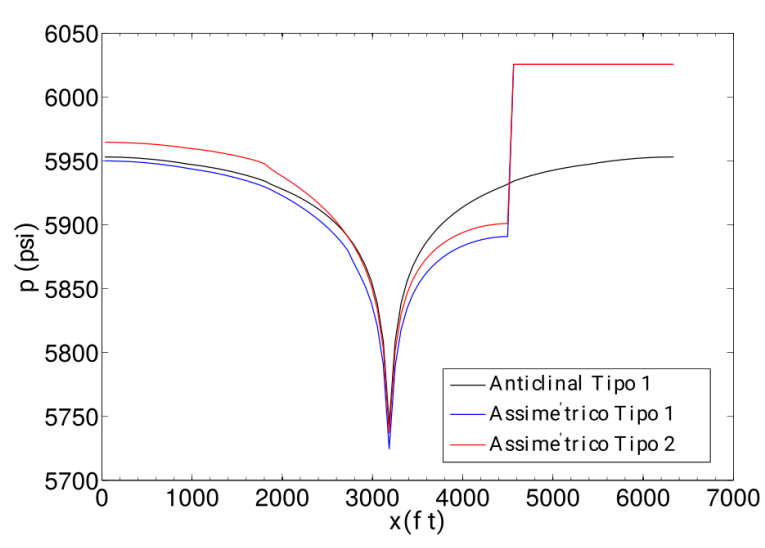

Figura 20: Pressão do reservatório: reservatórios anticlinal de Tipo 1 e assimétricos de Tipos 1 e 2

O comportamento envolvendo os reservatórios assimétricos é considerado na Fig. 20, na qual tem-se os resultados para a pressão no reservatório. Três casos foram analisados: o reservatório simétrico do Tipo 1 e dois assimétricos dos Tipos 1 e 2. Em se tratando dos reservatórios assimétricos, no Tipo 1 existem mais regiões ativas na parte inferior do reservatório, enquanto que no do Tipo 2 as regiões ativas são mais numerosas na parte superior do reservatório. Além disso, os dois reservatórios apresentam regiões inativas na camada de referência para a apresentação dos resultados, ou seja, na camada do centro.

Pode-se perceber, na Fig. 20, que a partir de $x=4.550 \mathrm{ft}$ a pressão permanece constante e é justamente nessa região onde se dá o início das regiões inativas. Outra variação importante pode ser percebida em $x=1.820 \mathrm{ft}$, com a ocorrência de uma queda brusca da pressão do reservatório assimétrico do Tipo 2 (nessa região há uma mudança na geometria das camadas significativa). As pressões das regiões inativas são representadas, no gráfico, com o valor da pressão inicial devido à hidrostática. A partir dos resultados analisados, concluiu-se que o simulador foi capaz de fornecer valores do campo de pressão sensiveis às alterações nas estruturas dos reservatórios anticlinais. Inclusive, é possível 
perceber que as diferentes assimetrias levam a curvas de pressão diferenciadas mesmo nas regiões nas quais os reservatórios são iguais, como pode ser observado para $x=2.000 \mathrm{ft}$.

\section{Conclusões}

Entende-se que a técnica específica para a representação de células inativas (módulo de rastreamento), objetivo principal deste trabalho, foi criada com sucesso para malhas uniformes, a partir de uma estratégia de busca que permite a definição de células com permeabilidades nulas e que não cria empecilhos para o refinamento de malha (as informações são baseadas na geometria das regiões inativas e não na indexação das células). Portando, considera-se que os efeitos inerentes à geometria do reservatório anticlinal foram incorporados e que os resultados obtidos foram promissores.

No que diz respeito ao desempenho computacional, a partir da paralelização do método dos Gradientes Conjugados usando o OpenMP, as performances alcançadas foram satisfatórias. A estagnação do speedup, a partir de um determinado número de threads, é conhecida na literatura e pode ser superada com o desenvolvimento de códigos numéricos ainda ainda mais elaborados (Chapman et al.; 2008) para que se consiga uma melhor distribuição de trabalho nas threads, embora esse não tenha sido um dos objetivos deste trabalho. Quanto à técnica de compressão CSR, o seu uso foi essencial. Porém, muito provavelmente ela poderia ser melhor aproveitada em uma estratégia na qual o sistema de equações leva-se em conta apenas as células ativas. O refinamento da malha também influenciou na determinação do speedup, conforme esperado, uma vez que o número de operações aritméticas cresce fortemente em função do número de células, aumentando o custo computacional significativamente.

Apesar da limitação do speedup máximo alcançado, em termos práticos houve uma redução significativa com relação ao tempo total de simulação, dos problemas que apresentam interesse na engenharia e que descrevem situações reais da produção de hidrocarbonetos em reservatórios do tipo anticlinal.

Quanto aos resultados da análise sensibilidade, considera-se que o comportamento físico esperado foi adequadamente capturado para as variações da permeabilidade, da compressibilidade e da vazão, e que os mesmos foram sensíveis às alterações na estrutura do reservatório anticlinal.

\section{Agradecimentos}

Os autores gostariam de agradecer ao suporte financeiro fornecido pela CAPES, CNPq e pela FAPERJ.

\section{Referências}

Abou-Kassem, J. H. and Ertekin, T. (1992). An efficient algorithm for removal of inactive blocks in reservoir simulation, Journal of Canadian Petroleum Technology 31(2): 25-31.
Abou-Kassem, J. H., Farouq Ali, S. M. and Islam, M. R. (2006). Petroleum Reservoir Simulation, A Basic Approach, Gulf Publishing Company, Houston,USA.

Al-Mohannadi, N. (2004). Simulation of Horizontal Well Tests, PhD thesis, Colorado School of Mines, Golden, USA.

Almahmoud, M., Khalil, M. and Moustafa, A. (2009). The Jinadriyah anticlines: a surface model for oil fields in eastern Saudi Arabia, Advances in Water Resources pp. 213-234.

Antunes Filho, A. and Xavier, A. C. C. (2015). Solução de sistemas lineares esparsos utilizando CUDA: uma comparação de desempenho em sistemas Windows e Linux, Revista de Ciências Exatas e Tecnologia 8(8): 181-195.

ARB (2011). OpenMP application program interface, v. 3.1, OpenMP Architecture Review Board .

Behzadinasab, M. (2015). Development and application of a parallel chemical compositional reservoir simulator, Dissertação de mestrado, The University of Texas at Austin, Texas.

Bourdet, D. (2002). Well Test Analysis: the use of advanced interpretation models, Elsevier, Amsterdam.

Brunschen, C. and Brorsson, M. (2000). OdinMP/CCp - a portable implementation of OpenMP for C., Concurrency - Practice and Experience 12(12): 11931203.

Canal, A. (2000). Paralelizaçao de Métodos de Resolução de Sistemas Lineares Esparsos com o DECK em um Cluster de PCs, PhD thesis, Universidade Federal do Rio Grande do Sul.

Chapman, B., Jost, G. and van der Pas, R. (2008). Using OpenMP: Portable Shared Memory Parallel Programming, Vol. 10, MIT press.

Eckert, A., Zhang, W., Liu, X. and Paradeis, M. (2014). Numerical estimates of the maximum sustainable pore pressure in anticline formations using the tensor based concept of pore pressure-stress coupling, Journal of Rock Mechanics and Geotechnical Engineering pp. 60-72.

Ertekin, T., Abou-Kassem, J. H. and King, G. R. (2001). Basic Applied Reservoir Simulation, SPE Textbook Series 7, Society of Petroleum Engineers, Richardson.

Grama, A., G., K., V., K. and A., G. (2003). Introduction to Parallel Computing, 2 edn, Pearson.

Guo, B., Lyons, W. C. and Ghalambor, A. (2007). Petroleum Production Engineering, A ComputerAssisted Approach, Elsevier Science \& Technology Books.

Hemstad, J. (2013). Modeling a non-uniform memory access architecture for optimizing conjugate gradient performance with sparse matrices, $\mathrm{PhD}$ thesis, College of Saint Benedict and Saint John's University.

Hestenes, M. and Stiefel, E. (1952). Methods of conjugate gradients for solving linear systems, Journal of Research of the National Bureau of Standards 49(6): 409-436. 
Nick, H. M., Raoof, A., Centler, F., Thullner, M. and Regnier, P. (2013). Reactive dispersive contaminant transport in coastal aquifers: Numerical simulation of a reactive Henry problem, Journal of Contaminant Hydrology 145: 90-104.

Oser, D. (2017). Petroleum geology, http://davidosergeology.tripod.com/petroleomgeology/id6.html.

Peaceman, D. W. (1983). Interpretation of well-block pressures in numerical reservoir simulation with nonsquare grid blocks and anisotropic permeability, Society of Petroleum Engineers Journal 23(3): 531-543.

Rosa, A. J., Carvalho, R. S. and Xavier, J. A. D. (2006). Engenharia de Reservatórios de Petróleo, Interciência, Rio de Janeiro, Brasil.

Souza, G. (2013). Acoplamento poço-reservatório na simulação numérica de reservatórios de gás, $\mathrm{PhD}$ thesis, Universidade Estadual do Norte Fluminense, Macaé, Brasil.

Torelli, J. and Bruno, O. (2004). Programação paralela em SMPs com OpenMP e POSIX Threads: um estudo comparativo, Anais do IV Congresso Brasileiro de Computaçao (CBComp), Vol. 1, pp. 486-491.

Venâncio Júnior, A. and Silva, R. (2004). Computação distribuída de ato desempenho - um estudo de caso de acesso móvel.

Werneck, L. F. (2016). Implementação paralelizada de métodos de resolução de sistemas algébricos na simulação de reservatórios de gás, Dissertação de mestrado, Universidade do Estado do Rio de Janeiro, Nova Friburgo.

Werneck, L. F., Freitas, M. M., Silva Júnior, H. G., Souza, G. and Souto, H. P. A. (2016). An OpenMP parallel implementation for numerical simulation of gas reservoirs using Intel Xeon Phi Coprocessor, Revista Interdisciplinar de Pesquisa em Engenharia 2(21): 37-56. 